\title{
FLOWS AND INVARIANCE FOR DEGENERATE ELLIPTIC OPERATORS
}

\section{A. F. M. TER ELST ${ }^{\bowtie}$, DEREK W. ROBINSON and ADAM SIKORA}

\author{
(Received 30 March 2009; accepted 18 February 2011)
}

Communicated by A. J. Price

\begin{abstract}
Let $S$ be a sub-Markovian semigroup on $L_{2}\left(\mathbb{R}^{d}\right)$ generated by a self-adjoint, second-order, divergenceform, elliptic operator $H$ with $W^{1, \infty}\left(\mathbb{R}^{d}\right)$ coefficients $c_{k l}$, and let $\Omega$ be an open subset of $\mathbb{R}^{d}$. We prove that if either $C_{c}^{\infty}\left(\mathbb{R}^{d}\right)$ is a core of the semigroup generator of the consistent semigroup on $L_{p}\left(\mathbb{R}^{d}\right)$ for some $p \in[1, \infty]$ or $\Omega$ has a locally Lipschitz boundary, then $S$ leaves $L_{2}(\Omega)$ invariant if and only if it is invariant under the flows generated by the vector fields $\sum_{l=1}^{d} c_{k l} \partial_{l}$ for all $k$. Further, for all $p \in[1,2]$ we derive sufficient conditions on the coefficients for the core property to be satisfied. Then by combination of these results we obtain various examples of invariance in terms of boundary degeneracy both for Lipschitz domains and domains with fractal boundaries.
\end{abstract}

2010 Mathematics subject classification: primary 35J70.

Keywords and phrases: degenerate elliptic operator, flow, invariance.

\section{Introduction}

Let $S$ be a sub-Markovian semigroup on $L_{2}\left(\mathbb{R}^{d}\right)$ generated by a self-adjoint secondorder elliptic operator $H$ in divergence form. If the operator is strongly elliptic then $S$ acts ergodically, that is, there are no nontrivial $S$-invariant subspaces of $L_{2}\left(\mathbb{R}^{d}\right)$. Nevertheless there are many examples of degenerate elliptic operators for which there are subspaces $L_{2}(\Omega)$ invariant under the action of $S$ (see, for example, [8, 10, 11, 25]). Our aim is to examine operators with coefficients which are Lipschitz continuous and characterize the $S$-invariance of $L_{2}(\Omega)$ by the invariance under a family of associated flows. In order to formulate our main result we need some further notation.

First, define the positive symmetric operator $H_{0}$ with domain $D\left(H_{0}\right)=C_{c}^{\infty}\left(\mathbb{R}^{d}\right)$ and action

$$
H_{0} \varphi=-\sum_{k, l=1}^{d} \partial_{k} c_{k l} \partial_{l} \varphi,
$$

Part of this work was supported by the Marsden Fund Council from Government funding, administered by the Royal Society of New Zealand.

(C) 2011 Australian Mathematical Publishing Association Inc. 1446-7887/2011 \$16.00 
where the coefficients $c_{k l}=c_{l k} \in W^{1, \infty}\left(\mathbb{R}^{d}\right)$ are real and $C=\left(c_{k l}\right)$ is a positive semidefinite matrix over $\mathbb{R}^{d}$. Then the corresponding quadratic form $h_{0}$, given by

$$
h_{0}(\varphi)=\sum_{k, l=1}^{d}\left(\partial_{k} \varphi, c_{k l} \partial_{l} \varphi\right)
$$

with domain $D\left(h_{0}\right)=C_{c}^{\infty}\left(\mathbb{R}^{d}\right)$, is closable. The closure $h=\bar{h}_{0}$ determines in a canonical manner a positive self-adjoint extension $H$ of $H_{0}$, the Friedrichs extension [13] (see, for example, [22, Section 124], or [19, Ch. VI]). The closed form $h$ is a Dirichlet form and the self-adjoint semigroup $S$ generated by $H$ is automatically sub-Markovian (for details on Dirichlet forms and sub-Markovian semigroups see [3, 14]). For all $p \in[1, \infty]$ and $t>0$, there exists a unique continuous operator $S_{t}^{(p)}$ on $L_{p}\left(\mathbb{R}^{d}\right)$ such that $S_{t}^{(p)} \varphi=S_{t} \varphi$ for all $\varphi \in L_{2}\left(\mathbb{R}^{d}\right) \cap L_{p}\left(\mathbb{R}^{d}\right)$. Then $S^{(p)}$ is a continuous contraction semigroup on $L_{p}\left(\mathbb{R}^{d}\right)$. Note that the continuity on $L_{\infty}\left(\mathbb{R}^{d}\right)$ is with respect to the weak* topology. Let $H_{p}$ denote the generator of $S^{(p)}$. We will refer to $H_{2}$ (or just $H$ ) as the degenerate elliptic operator with coefficients $\left(c_{k l}\right)$.

Second, if $b_{1}, \ldots, b_{d} \in W^{1, \infty}\left(\mathbb{R}^{d}\right)$ then the first-order partial differential operator

$$
\varphi \mapsto \sum_{k=1}^{d} b_{k} \partial_{k} \varphi-\frac{1}{2} \sum_{k=1}^{d}\left(\partial_{k} b_{k}\right) I,
$$

with domain $C_{c}^{\infty}\left(\mathbb{R}^{d}\right)$, is essentially skew-adjoint (see, for example, [23, Theorem 3.1]). Therefore the principal part is closable and generates a positive, continuous, oneparameter group on $L_{2}\left(\mathbb{R}^{d}\right)$. We refer to such a group as a flow. Specifically we are interested in the flows associated with the coefficients $\left(c_{k l}\right)$ of $H$. For all $k \in\{1, \ldots, d\}$, let $Y_{k}$ denote the $L_{2}$ closure of the first-order partial differential operator

$$
\varphi \mapsto \sum_{l=1}^{d} c_{k l} \partial_{l} \varphi
$$

with domain $C_{c}^{\infty}\left(\mathbb{R}^{d}\right)$. Then denote by $T^{(k)}$ the flow generated by $Y_{k}$. The operators $Y_{1}, \ldots, Y_{d}$ were used by Oleŭnik and Radkevič [20] to analyse hypoellipticity and subellipticity properties of degenerate elliptic operators $H$ with $C^{\infty}$ coefficients $\left(c_{k l}\right)$ (see [18] for a review of these and related results). We, however, use the flows to characterize the invariant subspaces of the semigroup generated by $H$.

THEOREM 1.1. Let $\Omega$ be a measurable subset of $\mathbb{R}^{d}$. Consider the following conditions.

(I) $S_{t} L_{2}(\Omega) \subseteq L_{2}(\Omega)$ for all $t>0$.

(II) $T_{t}^{(k)} L_{2}(\Omega)=L_{2}(\Omega)$ for all $k \in\{1, \ldots, d\}$ and $t \in \mathbb{R}$.

Then (I) implies (II). Moreover, if $C_{c}^{\infty}\left(\mathbb{R}^{d}\right)$ is a core of $H_{p}$ for some $p \in[1, \infty]$, or if $\Omega$ is an open set with locally Lipschitz boundary, then (I) and (II) are equivalent.

Recall that an open set $\Omega$ is defined to have locally Lipschitz boundary if, for every $y \in \partial \Omega$, there exist an isometry $\Psi: \mathbb{R}^{d} \rightarrow \mathbb{R}^{d}$, a real function $\tau \in W^{1, \infty}\left(\mathbb{R}^{d-1}\right)$ and $r>0$, 
such that

$$
\Omega \cap B_{y}(r)=\left\{\Psi\left(x_{1}, x^{\prime}\right):\left(x_{1}, x^{\prime}\right) \in \mathbb{R} \times \mathbb{R}^{d-1}, \tau\left(x^{\prime}\right)<x_{1}\right\} \cap B_{y}(r),
$$

where $B_{y}(r)=\left\{x \in \mathbb{R}^{d}:|x-y|<r\right\}$. Thus, in a neighbourhood of $y$, the boundary $\partial \Omega$ of $\Omega$ is the graph of a Lipschitz function $\tau$ and $\Omega$ lies on one side of it, up to an isometry $\Psi$.

The condition that $C_{c}^{\infty}\left(\mathbb{R}^{d}\right)$ is a core of $H_{p}$ can be phrased in a different way. If $p<\infty$, then it is equivalent to the statement that the operator $H_{0}$, viewed as an operator in $L_{p}\left(\mathbb{R}^{d}\right)$, has a unique extension that generates a strongly continuous semigroup (see [1, Theorem 1.33]). One also says that $H_{0}$ is $L_{p}$ unique. For more characterizations, see [7, Appendix A, Theorem 1.2].

We shall show in Section 2 that the flows extend consistently to continuous oneparameter groups on $L_{p}\left(\mathbb{R}^{d}\right)$. Although the invariance properties of Theorem 1.1 are stated for the subspaces $L_{2}(\Omega)$, these are equivalent to similar invariance properties of the subspaces $L_{p}(\Omega)$. For example, the $S$-invariance of $L_{2}(\Omega)$ and the sub-Markovian property of $S$ give

$$
S_{t}\left(L_{2}(\Omega) \cap L_{p}(\Omega)\right) \subset L_{2}(\Omega) \cap L_{p}\left(\mathbb{R}^{d}\right)=L_{2}(\Omega) \cap L_{p}(\Omega) .
$$

Therefore $S_{t}^{(p)} L_{p}(\Omega) \subseteq L_{p}(\Omega)$ by continuity. Similarly, the $S^{(p)}$-invariance of $L_{p}(\Omega)$ implies the $S$-invariance of $L_{2}(\Omega)$. The argument for the flows is identical.

There is another variant of the theorem that we first establish. For all $\psi \in C_{c}^{\infty}\left(\mathbb{R}^{d}\right)$, define $Y_{\psi}$ as the $L_{2}$ closure of the first-order partial differential operator

$$
\varphi \mapsto \sum_{k, l=1}^{d}\left(\partial_{k} \psi\right) c_{k l} \partial_{l} \varphi
$$

with domain $C_{c}^{\infty}\left(\mathbb{R}^{d}\right)$, and let $T^{\psi}$ be the associated flow. Then invariance of $L_{2}(\Omega)$ under the $T^{(k)}$ is equivalent to invariance under the family of flows $T^{\psi}$. More precisely, the following proposition holds.

Proposition 1.2. Let $\Omega$ be a measurable subset of $\mathbb{R}^{d}$. The following conditions are equivalent.

(I) $T_{t}^{\psi} L_{2}(\Omega)=L_{2}(\Omega)$ for all $\psi \in C_{c}^{\infty}\left(\mathbb{R}^{d}\right)$ and $t \in \mathbb{R}$.

(II) $T_{t}^{(k)} L_{2}(\Omega)=L_{2}(\Omega)$ for all $k \in\{1, \ldots, d\}$ and $t \in \mathbb{R}$.

This will be established in Section 2. Then we prove Theorem 1.1 in Section 3, and we derive various core criteria in Section 4. Our results are illustrated by several examples in Section 5.

\section{Flows}

In this section, we derive some properties of the flows defined in Section 1 and prove Proposition 1.2. Although we deal primarily with the flows on $L_{2}\left(\mathbb{R}^{d}\right)$, we will 
need some properties of their extensions to $L_{\infty}\left(\mathbb{R}^{d}\right)$ in Section 3. Therefore we begin by summarizing some general features of the flows.

Let $b_{1}, \ldots, b_{d} \in W^{1, \infty}\left(\mathbb{R}^{d}\right)$ and define $Y$ as the $L_{2}$ closure of the first-order differential operator $\varphi \mapsto \sum_{k=1}^{d} b_{k} \partial_{k} \varphi$ with domain $W^{1,2}\left(\mathbb{R}^{d}\right)$. Further, let $T$ denote the flow generated by $Y$. Then for all $p \in[1, \infty]$, the group $T$ leaves the subspace $L_{2}\left(\mathbb{R}^{d}\right) \cap L_{p}\left(\mathbb{R}^{d}\right)$ of $L_{2}\left(\mathbb{R}^{d}\right)$ invariant, and $T$ extends from $L_{2}\left(\mathbb{R}^{d}\right) \cap L_{p}\left(\mathbb{R}^{d}\right)$ to a flow $T^{[p]}$ on $L_{p}\left(\mathbb{R}^{d}\right)$ such that $T^{[p]}$ is strongly continuous if $p \in[1, \infty)$ and $T^{[\infty]}$ is weakly* continuous. The groups act in a consistent and compatible manner on the $L_{p}$ spaces. Moreover, $T^{[\infty]}$ is a group of automorphisms of $L_{\infty}\left(\mathbb{R}^{d}\right)$, that is,

$$
T_{t}^{[\infty]}(\psi \varphi)=\left(T_{t}^{[\infty]} \psi\right)\left(T_{t}^{[\infty]} \varphi\right)
$$

for all $\psi, \varphi \in L_{\infty}\left(\mathbb{R}^{d}\right)$ and $t \in \mathbb{R}$. Then since the $L_{\infty}$ functions are multipliers on the $L_{p}$ spaces, one deduces that

$$
T_{t}^{[p]}(\tau \varphi)=\left(T_{t}^{[\infty]} \tau\right)\left(T_{t}^{[p]} \varphi\right)
$$

for all $\tau \in L_{\infty}\left(\mathbb{R}^{d}\right), \varphi \in L_{p}\left(\mathbb{R}^{d}\right), p \in[1, \infty]$ and $t \in \mathbb{R}$. If $Y_{[p]}$ is the generator of $T^{[p]}$, then $W^{1, p}\left(\mathbb{R}^{d}\right) \subseteq D\left(Y_{[p]}\right)$ and $Y_{[p]} \varphi=\sum_{k=1}^{d} b_{k} \partial_{k} \varphi$ for all $\varphi \in W^{1, p}\left(\mathbb{R}^{d}\right)$.

These properties depend critically on the fact that $Y$ is a first-order partial differential operator with coefficients $b_{k} \in W^{1, \infty}\left(\mathbb{R}^{d}\right)$. They can be verified either by general arguments of functional analysis (see, for example, [24, Theorem V.4.1]) or by the methods of ordinary differential equations. The crucial observation in the latter context is that if $\varphi \in C_{c}^{\infty}\left(\mathbb{R}^{d}\right)$, then $\left(T_{t} \varphi\right)(x)=\varphi\left(\omega_{t}(x)\right)$, where $t \mapsto \omega_{t}(x)$ is the unique solution of the differential equation $(d / d t) \omega_{t}(x)=b\left(\omega_{t}(x)\right)$ with initial value $\omega_{0}(x)=x$ (see, for example, [16, Chs 2 and 3]). We write $\omega_{t}(x)=e^{t Y} x$.

Our first result is an approximation result that will be needed on $L_{2}\left(\mathbb{R}^{d}\right)$ but whose proof extends to the $L_{p}$ spaces.

Proposition 2.1. Let $p \in[1, \infty]$. Let $Y_{[p]}$ denote the generator of the flow $T^{[p]}$ on $L_{p}\left(\mathbb{R}^{d}\right)$. Further, let $\tau \in C_{c}^{\infty}\left(\mathbb{R}^{d}\right)$ with $\int \tau=1$ and for all $n \in \mathbb{N}$ define $\tau_{n} \in C_{c}^{\infty}\left(\mathbb{R}^{d}\right)$ by $\tau_{n}(x)=n^{d} \tau(n x)$. Then $\lim _{n \rightarrow \infty} Y_{[p]}\left(\tau_{n} * \varphi\right)=Y_{[p]} \varphi$ in $L_{p}\left(\mathbb{R}^{d}\right)$ for all $\varphi \in D\left(Y_{[p]}\right)$ if $p<\infty$. If $p=\infty$, then $\lim _{n \rightarrow \infty} Y_{[\infty]}\left(\tau_{n} * \varphi\right)=Y_{[\infty]} \varphi$ weakly* in $L_{\infty}\left(\mathbb{R}^{d}\right)$ for all $\varphi \in D\left(Y_{[\infty]}\right)$.

Proof. First, for all $n \in \mathbb{N}$ define the bounded operator $B_{n}: L_{p}\left(\mathbb{R}^{d}\right) \rightarrow L_{p}\left(\mathbb{R}^{d}\right)$ by

$$
B_{n} \varphi=\sum_{k=1}^{d} \tau_{n} *\left(\left(\partial_{k} b_{k}\right) \varphi\right)+\sum_{k=1}^{d} \int d y\left(\partial_{k} \tau_{n}\right)(y)\left(\left(I-L_{y}\right) b_{k}\right)\left(L_{y} \varphi\right)
$$

where $L$ denotes the left regular representation of $\mathbb{R}^{d}$, that is, $\left(L_{y} \psi\right)(x)=\psi(x-y)$. 
Second, if $\varphi \in C_{c}^{\infty}\left(\mathbb{R}^{d}\right)$ and $n \in \mathbb{N}$, then

$$
\begin{aligned}
Y_{[p]}\left(\tau_{n} * \varphi\right)= & \sum_{k=1}^{d} b_{k} \int d y \tau_{n}(y) L_{y} \partial_{k} \varphi \\
= & \sum_{k=1}^{d} \int d y \tau_{n}(y)\left(b_{k}-L_{y} b_{k}\right) L_{y} \partial_{k} \varphi \\
& +\sum_{k=1}^{d} b_{k} \int d y \tau_{n}(y) L_{y}\left(b_{k} \partial_{k} \varphi\right) .
\end{aligned}
$$

The second term is equal to $\tau_{n} * Y_{[p]} \varphi$. For the first term, use $L_{y} \partial_{k} \varphi=-\partial L_{y} \varphi / \partial y_{k}$. Then integration by parts gives

$$
Y_{[p]}\left(\tau_{n} * \varphi\right)-\tau_{n} * Y_{[p]} \varphi=\sum_{k=1}^{d} \int d y \frac{\partial}{\partial y_{k}}\left(\tau_{n}(y)\left(b_{k}-L_{y} b_{k}\right)\right)\left(L_{y} \varphi\right)=B_{n} \varphi .
$$

Since $B_{n}$ is bounded, one deduces by density that

$$
Y_{[p]}\left(\tau_{n} * \varphi\right)-\tau_{n} * Y_{[p]} \varphi=B_{n} \varphi
$$

for all $n \in \mathbb{N}$ and $\varphi \in D\left(Y_{[p]}\right)$.

Third, it follows from the definition of $B_{n}$ that

$$
\begin{aligned}
\left\|B_{n} \varphi\right\|_{p} & \leq \sum_{k=1}^{d}\left(\left\|\left(\partial_{k} b_{k}\right) \varphi\right\|_{p}+\int d y\left|\left(\partial_{k} \tau_{n}\right)(y)\right|\left\|\left(\left(I-L_{y}\right) b_{k}\right)\left(L_{y} \varphi\right)\right\|_{p}\right) \\
& \leq \sum_{k=1}^{d}\left\|b_{k}\right\|_{W^{1, \infty}\left(\mathbb{R}^{d}\right)}\|\varphi\|_{p}+\sum_{k=1}^{d} \int d y\left|\left(\partial_{k} \tau_{n}\right)(y)\right|\left\|\left(I-L_{y}\right) b_{k}\right\|_{\infty}\|\varphi\|_{p}
\end{aligned}
$$

for all $n \in \mathbb{N}$ and $\varphi \in L_{p}\left(\mathbb{R}^{d}\right)$. But

$$
\left\|\left(I-L_{y}\right) b_{k}\right\|_{\infty} \leq|y|\left\|b_{k}\right\|_{W^{1, \infty}\left(\mathbb{R}^{d}\right)}
$$

and

$$
\int d y\left|\left(\partial_{k} \tau_{n}\right)(y)\right||y|=\int d y\left|\left(\partial_{k} \tau\right)(y)\right||y| .
$$

Therefore $\left\|B_{n} \varphi\right\|_{p} \leq M\|\varphi\|_{p}$ uniformly for all $n \in \mathbb{N}$ and $\varphi \in L_{p}\left(\mathbb{R}^{d}\right)$, where

$$
M=\sum_{k=1}^{d}\left(1+\int d y\left|\left(\partial_{k} \tau\right)(y)\right||y|\right)\left\|b_{k}\right\|_{W^{1, \infty}\left(\mathbb{R}^{d}\right)} .
$$

The conclusion holds for all $p \in[1, \infty]$. So $B_{1}, B_{2}, \ldots$ are equicontinuous.

Next, assume that $p<\infty$. Then $\lim _{n \rightarrow \infty} \tau_{n} * \varphi=\varphi$ in $W^{1, p}\left(\mathbb{R}^{d}\right)$ for all $\varphi \in$ $W^{1, p}\left(\mathbb{R}^{d}\right)$. Consequently, $\lim _{n \rightarrow \infty} Y_{[p]}\left(\tau_{n} * \varphi\right)=Y_{[p]} \varphi$ strongly in $L_{p}\left(\mathbb{R}^{d}\right)$. Furthermore, $\lim _{n \rightarrow \infty} \tau_{n} *\left(Y_{[p]} \varphi\right)=Y_{[p]} \varphi$ strongly in $L_{p}\left(\mathbb{R}^{d}\right)$. Therefore it follows from (2.2) that $\lim _{n \rightarrow \infty} B_{n} \varphi=0$ in $L_{p}\left(\mathbb{R}^{d}\right)$ for all $\varphi \in W^{1, p}\left(\mathbb{R}^{d}\right)$. Since $W^{1, p}\left(\mathbb{R}^{d}\right)$ is strongly dense in $L_{p}\left(\mathbb{R}^{d}\right)$ and $B_{1}, B_{2}, \ldots$ are equicontinuous it follows that $\lim _{n \rightarrow \infty} B_{n} \varphi=0$ in $L_{p}\left(\mathbb{R}^{d}\right)$ 
for all $\varphi \in L_{p}\left(\mathbb{R}^{d}\right)$. Finally, let $\varphi \in D\left(Y_{[p]}\right)$. Then one establishes from (2.2) that

$$
\lim _{n \rightarrow \infty} Y_{[p]}\left(\tau_{n} * \varphi\right)=\lim _{n \rightarrow \infty}\left(\tau_{n} * Y_{[p]} \varphi+B_{n} \varphi\right)=Y_{[p]} \varphi
$$

in $L_{p}\left(\mathbb{R}^{d}\right)$.

The argument when $p=\infty$ is very similar. If $\varphi \in W^{1, \infty}\left(\mathbb{R}^{d}\right)$, then $\lim \tau_{n} * \varphi=\varphi$ and $\lim \partial_{k} \tau_{n} * \varphi=\partial_{k} \varphi$ weakly*. Therefore $\lim Y_{[\infty]}\left(\tau_{n} * \varphi\right)=Y_{[\infty]} \varphi$ weakly* on $L_{\infty}\left(\mathbb{R}^{d}\right)$. Then since $W^{1, \infty}\left(\mathbb{R}^{d}\right)$ is weakly* dense in $L_{\infty}\left(\mathbb{R}^{d}\right)$ and $B_{1}, B_{2}, \ldots$ are equicontinuous, the desired conclusion follows as before.

Now we return to consideration of the vector fields $Y_{1}, \ldots, Y_{d}$ defined in Section 1 acting on $L_{2}\left(\mathbb{R}^{d}\right)$.

Corollary 2.2. Let $\tau$ and $\tau_{n}$ be as in Proposition 2.1. Then for all $\varphi \in \bigcap_{k=1}^{d} D\left(Y_{k}\right)$, one has $\lim _{n \rightarrow \infty} Y_{k}\left(\tau_{n} * \varphi\right)=Y_{k} \varphi$ for all $k \in\{1, \ldots, d\}$. Therefore $W^{\infty, 2}\left(\mathbb{R}^{d}\right)$ is a simultaneous core of the $Y_{1}, \ldots, Y_{d}$.

The second statement of the corollary follows because convolution with $\tau_{n}$ maps $L_{2}\left(\mathbb{R}^{d}\right)$ into $W^{\infty, 2}\left(\mathbb{R}^{d}\right)$.

Now we turn to the proof of Proposition 1.2. Note that if $T$ is a flow with generator $Y$ then $T$-invariance of $L_{2}(\Omega)$ is equivalent to the commutation of $Y$ and the operator of multiplication with $\mathbb{1}_{\Omega}$, that is, if $\varphi \in D(Y)$ then $\mathbb{1}_{\Omega} \varphi \in D(Y)$ and $Y\left(\mathbb{1}_{\Omega} \varphi\right)=\mathbb{1}_{\Omega} Y \varphi$.

Proof of Proposition 1.2. First we show that (I) implies (II). Let $k \in\{1, \ldots, d\}$ and let $U \subset \mathbb{R}^{d}$ be a bounded open subset. There exist $\chi, \psi \in C_{c}^{\infty}\left(\mathbb{R}^{d}\right)$ such that $\left.\chi\right|_{U}=\mathbb{1}$ and $\psi(x)=x_{k}$ for all $x \in \operatorname{supp} \chi$. Then $Y_{k}(\chi \varphi)=Y_{\psi}(\chi \varphi)$ for all $\varphi \in C_{c}^{\infty}\left(\mathbb{R}^{d}\right)$. Since $\varphi \mapsto \chi \varphi$ is continuous on $D\left(Y_{k}\right)$ and on $D\left(Y_{\psi}\right)$ with the graph norm, it follows from Proposition 2.1 that $\chi \varphi \in D\left(Y_{k}\right)$ for all $\varphi \in D\left(Y_{\psi}\right)$. In particular, if $\varphi \in C_{c}^{\infty}\left(\mathbb{R}^{d}\right)$ with supp $\varphi \subseteq U$, then $\mathbb{1}_{\Omega} \varphi \in D\left(Y_{\psi}\right)$, and therefore $\mathbb{1}_{\Omega} \varphi=\chi \mathbb{1}_{\Omega} \varphi \in D\left(Y_{k}\right)$. Moreover,

$$
Y_{k}\left(\mathbb{1}_{\Omega} \varphi\right)=Y_{\psi}\left(\chi \mathbb{1}_{\Omega} \varphi\right)=\mathbb{1}_{\Omega} Y_{\psi}(\chi \varphi)=\mathbb{1}_{\Omega} Y_{k} \varphi .
$$

It follows by continuity that $\mathbb{1}_{\Omega} \varphi \in D\left(Y_{k}\right)$ and $Y_{k}\left(\mathbb{1}_{\Omega} \varphi\right)=\mathbb{1}_{\Omega} Y_{k} \varphi$ for all $\varphi \in D\left(Y_{k}\right)$. Therefore condition (II) is valid.

Now we show that (II) implies (I). It follows from condition (II) that $\mathbb{1}_{\Omega} \varphi \in D\left(Y_{k}\right)$ and $Y_{k}\left(\mathbb{1}_{\Omega} \varphi\right)=\mathbb{1}_{\Omega} Y_{k} \varphi$ for all $\varphi \in D\left(Y_{k}\right)$. Let $\psi \in C_{c}^{\infty}\left(\mathbb{R}^{d}\right)$. Then $Y_{\psi} \varphi=\sum_{k=1}^{d}\left(\partial_{k} \psi\right) Y_{k} \varphi$ for all $\varphi \in C_{c}^{\infty}\left(\mathbb{R}^{d}\right)$. Since the coefficients $c_{k l}$ are in $W^{1, \infty}\left(\mathbb{R}^{d}\right)$, it follows from Corollary 2.2 that $\varphi \in D\left(Y_{\psi}\right)$ and $Y_{\psi} \varphi=\sum_{k=1}^{d}\left(\partial_{k} \psi\right) Y_{k} \varphi$ for all $\varphi \in \bigcap_{k=1}^{d} D\left(Y_{k}\right)$. Hence if $\varphi \in C_{c}^{\infty}\left(\mathbb{R}^{d}\right)$, then $\mathbb{1}_{\Omega} \varphi \in D\left(Y_{\psi}\right)$ and $Y_{\psi}\left(\mathbb{1}_{\Omega} \varphi\right)=\mathbb{1}_{\Omega} Y_{\psi} \varphi$. By density, the latter formula extends to all $\varphi \in D\left(Y_{\psi}\right)$, and therefore condition (I) is valid.

Finally, we note that the flows $T^{\psi}$ can be defined for all $\psi \in W^{2, \infty}\left(\mathbb{R}^{d}\right)$, and the conditions of Proposition 1.2 are equivalent to invariance of $L_{2}(\Omega)$ for all $T_{t}^{\psi}$ with $\psi \in W^{2, \infty}\left(\mathbb{R}^{d}\right)$ and $t>0$. This follows from the arguments of the foregoing proof. 


\section{Semigroup invariance}

In this section, we prove Theorem 1.1. First, however, we observe that condition (II) of the theorem, that is, the invariance of $L_{2}(\Omega)$ under the flows $T^{(k)}$, is equivalent to the $T^{\psi}$-invariance of $L_{2}(\Omega)$ for all $\psi \in C_{c}^{\infty}\left(\mathbb{R}^{d}\right)$. This is a direct consequence of Proposition 1.2, which was established in the previous section. Therefore in the subsequent discussion we will consider the $T^{\psi}$-invariance condition.

Proof of Theorem 1.1. By the foregoing observation, to show that (I) implies (II), it suffices to prove the $T^{\psi}$-invariance of $L_{2}(\Omega)$ for all $\psi \in C_{c}^{\infty}\left(\mathbb{R}^{d}\right)$.

First, it follows from the density of $C_{c}^{\infty}\left(\mathbb{R}^{d}\right)$ in $D(h)$ that there exists a unique bilinear map $\Gamma: D(h) \times D(h) \rightarrow L_{1}\left(\mathbb{R}^{d}\right)$, the carré du champ, such that

$$
\Gamma(\psi, \varphi)=\sum_{k, l=1}^{d} c_{k l}\left(\partial_{k} \psi\right)\left(\partial_{l} \varphi\right)
$$

for all $\psi, \varphi \in W^{1,2}\left(\mathbb{R}^{d}\right)$. Then $\|\Gamma(\psi, \varphi)\|_{1} \leq h(\psi)^{1 / 2} h(\varphi)^{1 / 2}$ for all $\psi, \varphi \in D(h)$ by the Cauchy-Schwarz inequality. Moreover,

$$
\int \tau \Gamma(\psi, \varphi)=\frac{1}{2}(h(\tau \psi, \varphi)+h(\psi, \tau \varphi)-h(\tau, \psi \varphi))
$$

for all $\tau, \psi, \varphi \in C_{c}^{\infty}\left(\mathbb{R}^{d}\right)$. Then (3.1) extends to all $\tau, \psi, \varphi \in D(h) \cap L_{\infty}\left(\mathbb{R}^{d}\right)$ by density.

Second, the form $h$ is local in the sense that $h(\psi, \varphi)=0$ for all $\psi, \varphi \in D(h)$ such that $\psi \varphi=0$ (see [28]). It follows from (3.1) that $\Gamma$ is local in the same sense.

Third, since $L_{2}(\Omega)$ is $S$-invariant, the operation of multiplication by $\mathbb{1}_{\Omega}$ maps $D(h)$ into itself. Therefore, if $\psi, \varphi, \tau \in D(h) \cap L_{\infty}\left(\mathbb{R}^{d}\right)$, then $\mathbb{1}_{\Omega} \varphi, \mathbb{1}_{\Omega} \tau \in D(h) \cap L_{\infty}\left(\mathbb{R}^{d}\right)$. By locality of $h$, one deduces from (3.1) that

$$
\begin{aligned}
\int \tau \Gamma\left(\psi, \mathbb{1}_{\Omega} \varphi\right) & =\frac{1}{2}\left(h\left(\tau \psi, \mathbb{1}_{\Omega} \varphi\right)+h\left(\psi, \tau \mathbb{1}_{\Omega} \varphi\right)-h\left(\tau, \psi \mathbb{1}_{\Omega} \varphi\right)\right) \\
& =\frac{1}{2}\left(h\left(\mathbb{1}_{\Omega} \tau \psi, \varphi\right)+h\left(\psi, \mathbb{1}_{\Omega} \tau \varphi\right)-h\left(\mathbb{1}_{\Omega} \tau, \psi \varphi\right)\right) \\
& =\int \mathbb{1}_{\Omega} \tau \Gamma(\psi, \varphi) .
\end{aligned}
$$

Hence $\Gamma\left(\psi, \mathbb{1}_{\Omega} \varphi\right)=\mathbb{1}_{\Omega} \Gamma(\psi, \varphi)$. But $D(h) \cap L_{\infty}\left(\mathbb{R}^{d}\right)$ is dense in $D(h)$, and therefore $\Gamma\left(\psi, \mathbb{1}_{\Omega} \varphi\right)=\mathbb{1}_{\Omega} \Gamma(\psi, \varphi)$ for all $\psi, \varphi \in D(h)$.

Now fix $\psi \in C_{c}^{\infty}\left(\mathbb{R}^{d}\right)$, and let $\tau \in C_{c}^{\infty}\left(\mathbb{R}^{d}\right)$. Then

$$
\left(\left(Y_{\psi}\right)^{*} \tau, \eta\right)=\left(\tau, Y_{\psi} \eta\right)=(\tau, \Gamma(\psi, \eta))
$$

for all $\eta \in C_{c}^{\infty}\left(\mathbb{R}^{d}\right)$. One deduces that $\left(\left(Y_{\psi}\right)^{*} \tau, \eta\right)=(\tau, \Gamma(\psi, \eta))$ for all $\eta \in D(h)$, since $C_{c}^{\infty}\left(\mathbb{R}^{d}\right)$ is dense in $D(h)$. Choosing $\eta=\mathbb{1}_{\Omega} \varphi$, it follows that

$$
\begin{aligned}
\left(\left(Y_{\psi}\right)^{*} \tau, \mathbb{1}_{\Omega} \varphi\right) & =\left(\tau, \Gamma\left(\psi, \mathbb{1}_{\Omega} \varphi\right)\right)=\left(\mathbb{1}_{\Omega} \tau, \Gamma(\psi, \varphi)\right) \\
& =\left(\mathbb{1}_{\Omega} \tau, Y_{\psi} \varphi\right)=\left(\tau, \mathbb{1}_{\Omega} Y_{\psi} \varphi\right) .
\end{aligned}
$$


Since $C_{c}^{\infty}\left(\mathbb{R}^{d}\right)$ is a core of $\left(Y_{\psi}\right)^{*}$, one deduces that $\mathbb{1}_{\Omega} \varphi \in D\left(Y_{\psi}\right)$ and $Y_{\psi}\left(\mathbb{1}_{\Omega} \varphi\right)=\mathbb{1}_{\Omega} Y_{\psi} \varphi$. This conclusion then extends to all $\varphi \in D\left(Y_{\psi}\right)$ by density. Therefore $L_{2}(\Omega)$ is invariant under $T^{\psi}$.

To show that (II) implies (I), we have to consider two special cases.

Case 1. Let $p \in[1, \infty]$ and suppose that $C_{c}^{\infty}\left(\mathbb{R}^{d}\right)$ is a core of $H_{p}$.

Condition (II) is equivalent to the $T^{\psi}$ invariance of $L_{2}(\Omega)$ for all $\psi \in C_{c}^{\infty}\left(\mathbb{R}^{d}\right)$ by Proposition 1.2. Therefore we assume the latter condition.

Let $\psi, \tau \in C_{c}^{\infty}\left(\mathbb{R}^{d}\right)$. Then

$$
(H \psi, \tau \varphi)=\left(\tau, Y_{\psi} \varphi\right)+\left(\varphi, Y_{\psi} \tau\right)
$$

for all $\varphi \in C_{c}^{\infty}\left(\mathbb{R}^{d}\right)$. Since $C_{c}^{\infty}\left(\mathbb{R}^{d}\right)$ is dense in $D\left(Y_{\psi}\right)$, one deduces that

$$
(H \psi, \tau \varphi)=\left(\tau, Y_{\psi} \varphi\right)+\left(\varphi, Y_{\psi} \tau\right)
$$

for all $\varphi \in D\left(Y_{\psi}\right)$.

Now let $\psi, \tau, \varphi \in C_{c}^{\infty}\left(\mathbb{R}^{d}\right)$. Then by $T^{\psi}$-invariance of $L_{2}(\Omega)$ and (3.2), one deduces that $\mathbb{1}_{\Omega} \varphi \in D\left(Y_{\psi}\right)$ and

$$
\begin{aligned}
\left(H \psi, \tau \mathbb{1}_{\Omega} \varphi\right) & =\left(\tau, Y_{\psi}\left(\mathbb{1}_{\Omega} \varphi\right)\right)+\left(\mathbb{1}_{\Omega} \varphi, Y_{\psi} \tau\right) \\
& =\left(\mathbb{1}_{\Omega} \tau, Y_{\psi} \varphi\right)+\left(\mathbb{1}_{\Omega} \varphi, Y_{\psi} \tau\right) .
\end{aligned}
$$

Similarly

$$
\begin{aligned}
\left(\mathbb{1}_{\Omega} \psi, H(\tau \varphi)\right) & =\left(\mathbb{1}_{\Omega} \psi, Y_{\varphi}^{*} \tau\right)+\left(\mathbb{1}_{\Omega} \psi, Y_{\tau}^{*} \varphi\right) \\
& =\left(Y_{\varphi}\left(\mathbb{1}_{\Omega} \psi\right), \tau\right)+\left(Y_{\tau}\left(\mathbb{1}_{\Omega} \psi\right), \varphi\right) \\
& =\left(Y_{\varphi} \psi, \mathbb{1}_{\Omega} \tau\right)+\left(Y_{\tau} \psi, \mathbb{1}_{\Omega} \varphi\right)=\left(\mathbb{1}_{\Omega} \tau, Y_{\psi} \varphi\right)+\left(\mathbb{1}_{\Omega} \varphi, Y_{\psi} \tau\right)
\end{aligned}
$$

since $Y_{\varphi} \psi=Y_{\psi} \varphi$ and $Y_{\tau} \psi=Y_{\psi} \tau$. Therefore

$$
\left(H \psi, \mathbb{1}_{\Omega}(\tau \varphi)\right)=\left(\mathbb{1}_{\Omega} \psi, H(\tau \varphi)\right)
$$

for all $\psi, \tau, \varphi \in C_{c}^{\infty}\left(\mathbb{R}^{d}\right)$. Then since $C_{c}^{\infty}\left(\mathbb{R}^{d}\right)=\operatorname{span}\left(C_{c}^{\infty}\left(\mathbb{R}^{d}\right) \cdot C_{c}^{\infty}\left(\mathbb{R}^{d}\right)\right)$, one has

$$
\left(H \psi, \mathbb{1}_{\Omega} \varphi\right)=\left(\mathbb{1}_{\Omega} \psi, H \varphi\right)
$$

for all $\psi, \varphi \in C_{c}^{\infty}\left(\mathbb{R}^{d}\right)$. At this point we use the core assumption.

It follows from (3.3) by continuity that

$$
\left(H \psi, \mathbb{1}_{\Omega} \varphi\right)=\left(\mathbb{1}_{\Omega} \psi, H_{p} \varphi\right)
$$

for all $\psi \in C_{c}^{\infty}\left(\mathbb{R}^{d}\right)$ and all $\varphi \in D\left(H_{p}\right)$. But $\left|\left(H \psi, \mathbb{1}_{\Omega} \varphi\right)\right| \leq\|H \psi\|_{q}\|\varphi\|_{p}$, where $q$ is the dual variable to $p$. Therefore $\mathbb{1}_{\Omega} \psi \in D\left(H_{p}^{*}\right)=D\left(H_{q}\right)$ and

$$
H_{q}\left(\mathbb{1}_{\Omega} \psi\right)=\mathbb{1}_{\Omega} H \psi
$$

for all $\psi \in C_{c}^{\infty}\left(\mathbb{R}^{d}\right)$. But $\mathbb{1}_{\Omega} \psi \in L_{p}\left(\mathbb{R}^{d}\right) \cap L_{q}\left(\mathbb{R}^{d}\right)$, and furthermore $\mathbb{1}_{\Omega} \psi \in D\left(H_{q}\right)$ and $H_{q}\left(\mathbb{1}_{\Omega} \psi\right)=\mathbb{1}_{\Omega} H \psi \in L_{p}\left(\mathbb{R}^{d}\right)$. Hence it follows from the sub-Markovian property of the 
semigroup $S$ that $\mathbb{1}_{\Omega} \psi \in D\left(H_{p}\right)$ and

$$
H_{p}\left(\mathbb{1}_{\Omega} \psi\right)=\mathbb{1}_{\Omega} H \psi
$$

for all $\psi \in C_{c}^{\infty}\left(\mathbb{R}^{d}\right)$.

Finally, since $C_{c}^{\infty}\left(\mathbb{R}^{d}\right)$ is a core of $H_{p}$ it follows by continuity that $\mathbb{1}_{\Omega} \psi \in D\left(H_{p}\right)$ and

$$
H_{p}\left(\mathbb{1}_{\Omega} \psi\right)=\mathbb{1}_{\Omega} H_{p} \psi
$$

for all $\psi \in D\left(H_{p}\right)$. One immediately deduces that $S_{t}^{(p)}\left(\mathbb{1}_{\Omega} \psi\right)=\mathbb{1}_{\Omega} S_{t}^{(p)} \psi$ for all $t>0$ and $\psi \in L_{p}\left(\mathbb{R}^{d}\right)$. Therefore $L_{p}(\Omega)$ is $S^{(p)}$-invariant and $L_{2}(\Omega)$ is $S$-invariant by the discussion following Theorem 1.1.

Case 2. $\Omega$ is open and has a locally Lipschitz boundary.

Let $P_{\Omega}$ be the orthogonal projection of $L_{2}\left(\mathbb{R}^{d}\right)$ onto $L_{2}(\Omega)$. By assumption, $T^{\psi}$ leaves $L_{2}(\Omega)$ invariant for all $\psi \in C_{c}^{\infty}\left(\mathbb{R}^{d}\right)$. Hence

$$
T_{t}^{\psi} P_{\Omega}=P_{\Omega} T_{t}^{\psi} P_{\Omega}
$$

for all $t \in \mathbb{R}$. Let $B$ denote multiplication by the function $\sum_{k, l=1}^{d}\left(\partial_{k} \psi\right)\left(\partial_{l} c_{k l}\right)$ and set $M_{t}=e^{-t B}$ for all $t \in \mathbb{R}$. Clearly each $M_{t}$ leaves $L_{2}(\Omega)$ invariant. Therefore $L_{2}(\Omega)$ is left invariant by $\left(T_{-t / n}^{\psi} M_{-t / n}\right)^{n}$ for all $t \in \mathbb{R}$ and $n \in \mathbb{N}$. But $\left(Y_{\psi}\right)^{*}=-Y_{\psi}-B$. Then the Trotter product formula establishes that $\left(T_{t}^{\psi}\right)^{*}$ is the strong limit of $\left(T_{-t / n}^{\psi} M_{-t / n}\right)^{n}$ as $n \rightarrow \infty$. So $\left(T_{t}^{\psi}\right)^{*}$ leaves $L_{2}(\Omega)$ invariant. Hence $\left(T_{t}^{\psi}\right)^{*} P_{\Omega}=P_{\Omega}\left(T_{t}^{\psi}\right)^{*} P_{\Omega}$ for all $t \in \mathbb{R}$. Therefore $P_{\Omega} T_{t}^{\psi}=P_{\Omega} T_{t}^{\psi} P_{\Omega}$, and by (3.4) it follows that $T_{t}^{\psi} P_{\Omega}=P_{\Omega} T_{t}^{\psi}$ for all $t \in \mathbb{R}$. Then

$$
\mathbb{1}_{\Omega} T_{t}^{\psi} \varphi=P_{\Omega} T_{t}^{\psi} \varphi=T_{t}^{\psi} P_{\Omega} \varphi=T_{t}^{\psi}\left(\mathbb{1}_{\Omega} \varphi\right)=\left(T_{t}^{\psi[\infty]} \mathbb{1}_{\Omega}\right)\left(T_{t}^{\psi} \varphi\right)
$$

for all $\varphi \in C_{c}^{\infty}\left(\mathbb{R}^{d}\right)$ and $t \in \mathbb{R}$, where $T^{\psi[\infty]}$ denotes the extension of the flow $T^{\psi}$ to $L_{\infty}\left(\mathbb{R}^{d}\right)$ (see Section 2), and we have used (2.1). Since $T_{t}^{\psi}\left(C_{c}^{\infty}\left(\mathbb{R}^{d}\right)\right)$ is dense in $L_{2}\left(\mathbb{R}^{d}\right)$, one deduces that $T_{t}^{\psi[\infty]} \mathbb{1}_{\Omega}=\mathbb{1}_{\Omega}$ for all $t \in \mathbb{R}$.

Next let $\varphi \in C_{c}^{\infty}\left(\mathbb{R}^{d}\right)$. Then $\left(Y_{\psi}\right)^{*} \varphi \in L_{1}\left(\mathbb{R}^{d}\right) \cap L_{2}\left(\mathbb{R}^{d}\right)$, so $\left(Y_{\psi}^{[\infty]}\right)^{*} \varphi=\left(Y_{\psi}\right)^{*} \varphi$, where $Y_{\psi}^{[\infty]}$ is the generator of $T^{\psi[\infty]}$. Since

$$
\left(\left(T_{t}^{\psi[\infty]}\right)^{*} \varphi, \mathbb{1}_{\Omega}\right)=\left(\varphi, T_{t}^{\psi[\infty]} \mathbb{1}_{\Omega}\right)=\left(\varphi, \mathbb{1}_{\Omega}\right)
$$

for all $t \in \mathbb{R}$, it follows by differentiation that $\left(\left(Y_{\psi}\right)^{*} \varphi, \mathbb{1}_{\Omega}\right)=0$. Therefore, setting $\Phi_{k}=\sum_{l=1}^{d} c_{k l} \partial_{l} \psi$ for all $k \in\{1, \ldots, d\}$, one has

$$
\int_{\Omega} \operatorname{div}(\varphi \Phi)=\left(\left(Y_{\psi}\right)^{*} \varphi, \mathbb{1}_{\Omega}\right)=0 .
$$

At this point we use the Gauss-Green theorem, which is valid for open sets $\Omega$ with locally Lipschitz boundary (see, for example, [12, p. 209]). It states that

$$
\int_{\Omega} \operatorname{div} \Psi=\int_{\partial \Omega} d S\langle n, \Psi\rangle
$$

for all $\Psi \in W^{1, \infty}\left(\mathbb{R}^{d}\right)$ with compact support, where $\langle\cdot, \cdot\rangle$ denotes the inner product on $\mathbb{R}^{d}, d S$ is the Euclidean measure on $\partial \Omega$ and $n$ is the unit outward normal to $\partial \Omega$. 
The normal is defined $d S$ almost everywhere. Thus if one sets $\Psi=\varphi \Phi$ with $\varphi \in$ $C_{c}^{\infty}\left(\mathbb{R}^{d}\right)$, one has

$$
\int_{\Omega} \operatorname{div}(\varphi \Phi)=\int_{\partial \Omega} d S \varphi\langle n, \Phi\rangle=0,
$$

where the last equality uses (3.5). Since this is valid for all $\varphi \in C_{c}^{\infty}\left(\mathbb{R}^{d}\right)$, it follows that $\langle n, \Phi\rangle=0$ almost everywhere on $\partial \Omega$. So $\left\langle(\nabla \psi)(x), C(x) n_{x}\right\rangle=0$ for almost every $x \in \partial \Omega$. But this is also valid for all $\psi \in C_{c}^{\infty}\left(\mathbb{R}^{d}\right)$. Hence one must have $C(x) n_{x}=0$ for almost every $x \in \partial \Omega$. This corresponds to the condition of zero flux across the boundary as defined in [25], and then the $S$-invariance of $L_{2}(\Omega)$ follows from Theorem 1.2 of this reference.

The argument in [25] that zero flux implies invariance is somewhat indirect, as it first proves that the capacity of $\partial \Omega$ with respect to $h$ is zero and then uses this to deduce the $S$-invariance of $L_{2}(\Omega)$. Nevertheless, the same reasoning can be adapted to give a direct proof of the invariance, since the proof can be reduced to a local estimate as in [25]. The latter proof and this proof are an adaptation of the argument used to prove [11, Proposition 6.5].

First, it suffices to prove that if $\varphi \in C_{c}^{\infty}\left(\mathbb{R}^{d}\right)$ then $\mathbb{1}_{\Omega} \varphi \in D(h)$. This is a consequence of [8, Proposition 2.1] and locality of $h$. But this is obvious if the support of $\varphi$ and the boundary are disjoint. Therefore it suffices to consider $\varphi$ with support close to the boundary $\partial \Omega$. Then, however, one can use a decomposition of the identity to reduce to the case where supp $\varphi \subseteq B_{y}(r)$ with $y \in \partial \Omega$ and $r>0$ small.

Second, let $\tau, \Psi$ be as in (1.1). Without loss of generality we may assume that $\Psi(x)=x$ for all $x \in \mathbb{R}^{d}$. For all $n \in \mathbb{N}$ define $\psi_{n}: \mathbb{R}^{d} \rightarrow \mathbb{R}$ by $\psi_{n}(x)=\chi_{n}\left(x_{1}-\tau\left(x^{\prime}\right)\right)$, where $x=\left(x_{1}, x^{\prime}\right) \in \mathbb{R} \times \mathbb{R}^{d-1}$ and $\chi_{n}: \mathbb{R} \rightarrow \mathbb{R}$ is defined by

$$
\chi_{n}(t)= \begin{cases}0 & \text { if } t \leq 1 / n \\ \log (t n) / \log n & \text { if } 1 / n<t<1 \\ 1 & \text { if } t \geq 1\end{cases}
$$

Then $\lim \left(\psi_{n} \varphi\right)=\mathbb{1}_{\Omega} \varphi$ in $L_{2}\left(\mathbb{R}^{d}\right)$. Thus to establish that $\mathbb{1}_{\Omega} \varphi \in D(h)$, it suffices to prove that $\left\{h\left(\psi_{n} \varphi\right): n \in \mathbb{N}\right\}$ is bounded. But

$$
\begin{aligned}
h\left(\psi_{n} \varphi\right) & \leq 2 h(\varphi)+2 \int|\varphi|^{2} \sum_{k, l=1}^{d} c_{k l}\left(\partial_{k} \psi_{n}\right)\left(\partial_{l} \psi_{n}\right) \\
& \leq 2 h(\varphi)+2(\log n)^{-2} \int_{\mathbb{R}^{d-1}} d x^{\prime} \int_{\tau\left(x^{\prime}\right)+1 / n}^{\tau\left(x^{\prime}\right)+1} d x_{1}|\varphi(x)|^{2} \frac{\left\langle v_{x}, C(x) v_{x}\right\rangle}{\left(x_{1}-\tau\left(x^{\prime}\right)\right)^{2}}
\end{aligned}
$$

for all $n \in \mathbb{N}$, where $v_{x}=\left(1,-(\nabla \tau)\left(x^{\prime}\right)\right)$. Since the coefficients $c_{k l}$ are in $W^{1, \infty}\left(\mathbb{R}^{d}\right)$, there exists $M>0$ such that

$$
|\langle\xi, C(x) \xi\rangle-\langle\xi, C(z) \xi\rangle| \leq M\|\xi\|^{2}|x-z|
$$


for all $x, z, \xi \in \mathbb{R}^{d}$. If $x=\left(x_{1}, x^{\prime}\right) \in B_{y}(r)$, the function $\tau$ is differentiable at $x^{\prime}$ and $x_{1}=\tau\left(x^{\prime}\right)$, then

$$
\left\langle v_{x}, C\left(\tau\left(x^{\prime}\right), x^{\prime}\right) v_{x}\right\rangle=\left(1+\left|(\nabla \tau)\left(x^{\prime}\right)\right|^{2}\right)\left\langle n_{x}, C\left(\tau\left(x^{\prime}\right), x^{\prime}\right) n_{x}\right\rangle=0
$$

by the zero flux condition. It follows that $\left\langle v_{x}, C\left(x_{1}, x^{\prime}\right) v_{x}\right\rangle \leq M_{1}\left|x_{1}-\tau\left(x^{\prime}\right)\right|$ for all $x=\left(x_{1}, x^{\prime}\right) \in B_{y}(r)$ with $\tau$ differentiable at $x^{\prime}$, where $M_{1}=M\left(1+\|\nabla \tau\|_{\infty}\right)^{2}$. Hence

$$
\begin{gathered}
(\log n)^{-2} \int_{\mathbb{R}^{d-1}} d x^{\prime} \int_{\tau\left(x^{\prime}\right)+1 / n}^{\tau\left(x^{\prime}\right)+1} d x_{1}\left|\varphi\left(x_{1}, x^{\prime}\right)\right|^{2} \frac{\left\langle v_{x}, C\left(x_{1}, x^{\prime}\right) v_{x}\right\rangle}{\left(x_{1}-\tau\left(x^{\prime}\right)\right)^{2}} \\
\leq M_{1}(\log n)^{-2} \int_{\mathbb{R}^{d-1}} d x^{\prime} \int_{\tau\left(x^{\prime}\right)+1 / n}^{\tau\left(x^{\prime}\right)+1} d x_{1} \frac{\left|\varphi\left(x_{1}, x^{\prime}\right)\right|^{2}}{\left(x_{1}-\tau\left(x^{\prime}\right)\right)} \\
\leq M_{1}(\log n)^{-1}\|\varphi\|_{\infty}^{2}\left|K^{\prime}\right|
\end{gathered}
$$

uniformly for all $n \in \mathbb{N}$, where $K^{\prime} \subset \mathbb{R}^{d-1}$ is a compact set such that $\operatorname{supp} \varphi \subset \mathbb{R} \times K^{\prime}$. So $\left\{h\left(\psi_{n} \varphi\right): n \in \mathbb{N}\right\}$ is bounded, as required. In fact a slightly more detailed argument establishes that $\lim _{n \rightarrow \infty} h\left(\psi_{n} \varphi-\mathbb{1}_{\Omega} \varphi\right)=0$.

\section{Core properties}

In this section, we examine conditions that ensure that $C_{c}^{\infty}\left(\mathbb{R}^{d}\right)$ is a core for the degenerate elliptic operator $H$. Recall that $H$ was defined in Section 1 as the Friedrichs extension of $H_{0}$ with domain $D\left(H_{0}\right)=C_{c}^{\infty}\left(\mathbb{R}^{d}\right)$. Therefore $H$ generates a symmetric sub-Markovian semigroup $S$. We discuss criteria for $C_{c}^{\infty}\left(\mathbb{R}^{d}\right)$ to be a core of $H_{p}$, the generator of the extension $S^{(p)}$ of $S$ to $L_{p}\left(\mathbb{R}^{d}\right)$, in order to apply Theorem 1.1 to establish invariance properties.

First, we recall two known criteria for $C_{c}^{\infty}\left(\mathbb{R}^{d}\right)$ to be a core of $H\left(=H_{2}\right)$.

Proposition 4.1. If one of the following conditions is valid, then $C_{c}^{\infty}\left(\mathbb{R}^{d}\right)$ is a core of $H$.

(I) The matrix $\left(c_{k l}(x)\right)$ is invertible for each $x \in \mathbb{R}^{d}$.

(II) $c_{k l} \in W^{2, \infty}\left(\mathbb{R}^{d}\right)$ for all $k, l \in\{1, \ldots, d\}$.

Proof. If condition (I) is valid, then $C_{c}^{\infty}\left(\mathbb{R}^{d}\right)$ is a core, by the arguments in $[5$, Theorem 3.1]. Davies requires that the coefficients are smooth, but if the coefficients are bounded, then the smoothness condition can be relaxed to $W^{1, \infty}\left(\mathbb{R}^{d}\right)$. (See also the proof of Theorem 4.8.)

If condition (II) is valid, then $C_{c}^{\infty}\left(\mathbb{R}^{d}\right)$ is a core by [23, Section 6], or [9, Proposition 2.3], or by an adaptation of the proof of Proposition 2.1 to second-order operators.

Note that condition (I) is equivalent to the requirement that $\mu_{C}(x)>0$ for each $x \in \mathbb{R}^{d}$, where $\mu_{C}(x)$ is the smallest eigenvalue of $C(x)=\left(c_{k l}(x)\right)$. This is a condition of nondegeneracy that corresponds to strict ellipticity of $H_{0}$. But condition (II) imposes no such requirement. Indeed if the $c_{k l} \in W^{2, \infty}\left(\mathbb{R}^{d}\right)$, then one may even have $\mu_{C}(x)=0$ for all $x \in \mathbb{R}^{d}$. 
ExAmple 4.2. If $X_{1}=\partial_{1}$ and $X_{2}=\cos x_{1} \partial_{2}+\sin x_{1} \partial_{3}$, then the coefficient matrix $C(x)$ of the operator $H_{0}=-X_{1}^{2}-X_{2}^{2}$ on $C_{c}^{\infty}\left(\mathbb{R}^{3}\right)$ has eigenvalues $(1,1,0)$ independent of $x$. Therefore $C(x)$ is not zero and not invertible for any $x \in \mathbb{R}^{3}$. Nevertheless the elliptic operator $H_{0}$ has $C^{\infty}$ coefficients, is hypoelliptic and is essentially self-adjoint. It is a sub-Laplacian of the Euclidean motion group. See [6, Example II.5.1].

Our first aim is to establish a core result, Theorem 4.6, which involves a mixture of the two conditions of Proposition 4.1. It establishes that the $W^{2, \infty}$ condition is only necessary in the neighbourhood of the degeneracy set of the operator. Then we discuss an alternative version with the differentiability condition replaced by a boundedness property.

Lemma 4.3. If $\chi \in W^{2, \infty}\left(\mathbb{R}^{d}\right)$ and $\varphi \in D(H)$ then $\chi \varphi \in D(H)$.

Proof. Fix $\chi \in W^{2, \infty}\left(\mathbb{R}^{d}\right)$. Then it follows from [10, Lemma 3.4] that $\chi \varphi \in D(h)$ and

$$
h(\chi \varphi)^{1 / 2} \leq\|\chi\|_{\infty} h(\varphi)^{1 / 2}+\|\Gamma(\chi)\|_{\infty}^{1 / 2}\|\varphi\|_{2}
$$

for all $\varphi \in D(h)$, where for all $\tau \in W^{1, \infty}\left(\mathbb{R}^{d}\right)$ we define

$$
\Gamma(\tau)=\sum_{k, l=1}^{d} c_{k l}\left(\partial_{k} \tau\right)\left(\partial_{l} \tau\right) \in L_{\infty}\left(\mathbb{R}^{d}\right)
$$

If $\varphi, \psi \in C_{c}^{\infty}\left(\mathbb{R}^{d}\right)$, then

$$
h(\psi, \chi \varphi)=h(\chi \psi, \varphi)-\sum_{k, l=1}^{d} \int \psi \varphi\left(\partial_{k} c_{k l} \partial_{l} \chi\right)-2 \sum_{k, l=1}^{d} \int c_{k l}\left(\partial_{k} \varphi\right)\left(\partial_{l} \chi\right) \psi .
$$

So

$$
|h(\psi, \chi \varphi)| \leq|h(\chi \psi, \varphi)|+a\|\psi\|_{2}\|\varphi\|_{2}+2 h(\varphi)^{1 / 2}\|\Gamma(\chi)\|_{\infty}^{1 / 2}\|\psi\|_{2},
$$

where $a=\left\|\sum \partial_{k} c_{k l} \partial_{l} \chi\right\|_{\infty}$. Then, by continuity, (4.2) is valid for all $\psi, \varphi \in D(h)$. Finally, if $\varphi \in D(H)$, then

$$
|h(\chi \psi, \varphi)|=|(\chi \psi, H \varphi)| \leq\|H \varphi\|_{2}\|\chi\|_{\infty}\|\psi\|_{2}
$$

for all $\psi \in D(h)$. Using (4.2), it follows that $|h(\psi, \chi \varphi)| \leq c\|\psi\|_{2}$ for all $\psi \in D(h)$ for some $c>0$. Therefore $\chi \varphi \in D(H)$.

If $\emptyset \neq A \subseteq \mathbb{R}^{d}$ and $\delta>0$, then define $A_{\delta}=\left\{x \in \mathbb{R}^{d}: d_{A}(x)<\delta\right\}$, where $d_{A}(x)$ denotes the Euclidean distance from $x$ to $A$. Then $A_{\delta}$ is an open subset of $\mathbb{R}^{d}$.

LeMma 4.4. Let $H^{(1)}$ and $H^{(2)}$ be degenerate elliptic operators with coefficients $\left(c_{k l}^{(1)}\right)$ and $\left(c_{k l}^{(2)}\right)$ in $W^{1, \infty}\left(\mathbb{R}^{d}\right)$, and let $h^{(1)}$ and $h^{(2)}$ be the corresponding quadratic forms. Let $U \subseteq \mathbb{R}^{d}$ be an open set and suppose that $\left.c_{k l}^{(1)}\right|_{U}=\left.c_{k l}^{(2)}\right|_{U}$ for all $k, l \in\{1, \ldots, d\}$. Let $\varphi \in L_{2}\left(\mathbb{R}^{d}\right) \backslash\{0\}$ and $\delta>0$, and suppose that $(\operatorname{supp} \varphi)_{\delta} \subseteq U$.

Then $\varphi \in D\left(h^{(1)}\right)$ if and only if $\varphi \in D\left(h^{(2)}\right)$. Moreover, if these conditions are satisfied, then $h^{(1)}(\varphi)=h^{(2)}(\varphi)$. Similarly, $\varphi \in D\left(H^{(1)}\right)$ if and only if $\varphi \in D\left(H^{(2)}\right)$ and then $H^{(1)} \varphi=H^{(2)} \varphi$. Moreover, $\operatorname{supp} H^{(1)} \varphi \subseteq \operatorname{supp} \varphi$. 
Proof. There exists $\chi \in W^{2, \infty}\left(\mathbb{R}^{d}\right)$ such that $\left.\chi\right|_{\operatorname{supp} \varphi}=\mathbb{1}$ and supp $\chi \subseteq U$. Suppose that $\varphi \in D\left(h^{(1)}\right)$. Then there exists a sequence $\varphi_{1}, \varphi_{2}, \ldots \in W^{1,2}\left(\mathbb{R}^{d}\right)$ such that $\lim \varphi_{n}=\varphi$ in $D\left(h^{(1)}\right)$. Then $\lim \varphi_{n}=\varphi$ in $L_{2}\left(\mathbb{R}^{d}\right)$. But $h^{(1)}\left(\chi \varphi_{n}\right)=h^{(2)}\left(\chi \varphi_{n}\right)$ and

$$
h^{(1)}\left(\chi \varphi_{n}-\chi \varphi_{m}\right)=h^{(2)}\left(\chi \varphi_{n}-\chi \varphi_{m}\right)
$$

for all $n, m \in \mathbb{N}$. Therefore $\chi \varphi_{1}, \chi \varphi_{2}, \ldots$ is a Cauchy sequence in $D\left(h^{(2)}\right)$. Since $\lim \chi \varphi_{n}=\varphi$ in $L_{2}\left(\mathbb{R}^{d}\right)$, one deduces that $\varphi \in D\left(h^{(2)}\right)$ and $h^{(2)}(\varphi)=h^{(1)}(\varphi)$.

Finally, suppose that $\varphi \in D\left(H^{(1)}\right)$. If $\psi \in C_{c}^{\infty}\left(\mathbb{R}^{d}\right)$ and $\operatorname{supp} \psi \subseteq(\operatorname{supp} \varphi)^{\mathrm{c}}$, then

$$
\left(H^{(1)} \varphi, \psi\right)=h^{(1)}(\varphi, \psi)=0
$$

by locality. Therefore supp $H^{(1)} \varphi \subseteq \operatorname{supp} \varphi$. Clearly $\varphi \in D\left(h^{(1)}\right)$ and, by the first part, $\varphi \in D\left(h^{(2)}\right)$ too. Let $\psi \in D\left(h^{(2)}\right)$. Then $\chi \psi \in D\left(h^{(2)}\right)$ and $\operatorname{supp} \chi \psi \subseteq U$. Therefore $\chi \psi \in D\left(h^{(1)}\right)$. Then by locality, one deduces that

$$
h^{(2)}(\varphi, \psi)=h^{(2)}(\varphi, \chi \psi)+h^{(2)}(\varphi,(\mathbb{1}-\chi) \psi)=h^{(2)}(\varphi, \chi \psi)=h^{(1)}(\varphi, \chi \psi) .
$$

So

$$
\left|h^{(2)}(\varphi, \psi)\right|=\left|h^{(1)}(\varphi, \chi \psi)\right|=\left|\left(H^{(1)} \varphi, \chi \psi\right)\right| \leq\left\|H^{(1)} \varphi\right\|_{2}\|\chi\|_{\infty}\|\psi\|_{2} .
$$

Therefore $\varphi \in D\left(H^{(2)}\right)$. If $\psi \in C_{c}^{\infty}(U)$, then

$$
\left(H^{(1)} \varphi, \psi\right)=\left(\varphi, H^{(1)} \psi\right)=\left(\varphi, H^{(2)} \psi\right)=\left(H^{(2)} \varphi, \psi\right) .
$$

Since supp $H^{(1)} \varphi \subseteq U$ and $\operatorname{supp} H^{(2)} \varphi \subseteq U$, it follows that $H^{(1)} \varphi=H^{(2)} \varphi$.

Proposition 4.5. Let $A \subseteq \mathbb{R}^{d}$ and $\delta>0$. Further, let $H^{(1)}$ and $H^{(2)}$ be degenerate elliptic operators with $W^{1, \infty}$ coefficients $\left(c_{k l}^{(1)}\right)$ and $\left(c_{k l}^{(2)}\right)$. Suppose that $\emptyset \neq A \neq \mathbb{R}^{d}$, $\left.c_{k l}^{(1)}\right|_{A_{\delta}}=\left.c_{k l}\right|_{A_{\delta}}$ and $\left.c_{k l}^{(2)}\right|_{\left(A^{c}\right)_{\delta}}=\left.c_{k l}\right|_{\left(A^{c}\right)_{\delta}}$ for all $k, l \in\{1, \ldots, d\}$, and that $C_{c}^{\infty}\left(\mathbb{R}^{d}\right)$ is a core of both $H^{(1)}$ and $H^{(2)}$. Then $C_{c}^{\infty}\left(\mathbb{R}^{d}\right)$ is a core of $H$.

Proof. Let $\tau \in C_{c}^{\infty}\left(\mathbb{R}^{d}\right)$ be such that $\int \tau=1$ and $\tau(x)=0$ for all $x \in \mathbb{R}^{d}$ for which $|x|>$ $\delta / 4$. Let $\chi=\tau * \mathbb{1}_{A_{\delta / 2}}$. Then $\chi \in W^{2, \infty}\left(\mathbb{R}^{d}\right),\left.\chi\right|_{A_{\delta / 4}}=\mathbb{1}$ and supp $\chi \subseteq A_{3 \delta / 4}$. Moreover, $\operatorname{supp}(\mathbb{1}-\chi) \subseteq\left(A_{\delta / 4}\right)^{\mathrm{c}} \subseteq A^{\mathrm{c}}$. There exist $\chi_{1}, \chi_{2} \in W^{\infty, \infty}\left(\mathbb{R}^{d}\right)$ such that $\left.\chi_{1}\right|_{A_{3 \delta / 4}}=\mathbb{1}$, $\operatorname{supp} \chi_{1} \subseteq A_{\delta},\left.\chi_{2}\right|_{A^{\mathrm{c}}}=\mathbb{1}$ and $\operatorname{supp} \chi_{2} \subseteq\left(A^{\mathrm{c}}\right)_{\delta}$.

Let $\varphi \in D(H)$. It follows from Lemma 4.3 that $\chi \varphi \in D(H)$, and consequently $(\mathbb{1}-\chi) \varphi \in D(H)$. We shall show that we can approximate both elements by $C_{c}^{\infty}$ functions. We may assume that $\chi \varphi \neq 0 \neq(\mathbb{1}-\chi) \varphi$. Since $\operatorname{supp}(\chi \varphi) \subseteq A_{3 \delta / 4}$, one deduces from Lemma 4.4 that $\chi \varphi \in D\left(H^{(1)}\right)$ and $H^{(1)}(\chi \varphi)=H(\chi \varphi)$. By assumption, there exist $\varphi_{1}, \varphi_{2}, \ldots \in C_{c}^{\infty}\left(\mathbb{R}^{d}\right)$ such that $\lim \varphi_{n}=\chi \varphi$ in $D\left(H^{(1)}\right)$. Then $\lim \chi_{1} \varphi_{n}=$ $\chi_{1} \chi \varphi=\chi \varphi$ in $D\left(H^{(1)}\right)$ by Lemma 4.3. But $\chi_{1} \varphi_{n} \in C_{c}^{\infty}\left(\mathbb{R}^{d}\right)$ and $\operatorname{supp} \chi_{1} \varphi_{n} \subseteq A_{\delta}$ for all $n \in \mathbb{N}$. Therefore $\chi_{1} \varphi_{n} \in D(H)$ and $H\left(\chi_{1} \varphi_{n}\right)=H^{(1)}\left(\chi_{1} \varphi_{n}\right)$, again by Lemma 4.4. So $\lim \chi_{1} \varphi_{n}=\chi \varphi$ in $D(H)$. Similarly, using $H^{(2)}$ and $\chi_{2}$, there exists a sequence $\psi_{1}, \psi_{2}, \ldots \in C_{c}^{\infty}\left(\mathbb{R}^{d}\right)$ such that $\lim \chi_{2} \psi_{n}=(\mathbb{1}-\chi) \varphi$ in $D(H)$. Then $\lim \left(\chi_{1} \varphi_{n}+\right.$ $\left.\chi_{2} \psi_{n}\right)=\varphi$ in $D(H)$. Since $\chi_{1} \varphi_{n}+\chi_{2} \psi_{n} \in C_{c}^{\infty}\left(\mathbb{R}^{d}\right)$ the proposition follows. 
THEOREM 4.6. Suppose that there exist a set $A$ and $\delta>0$ such that $\emptyset \neq A \neq \mathbb{R}^{d}$, the matrix $\left(c_{k l}(x)\right)$ is invertible for each $x \in\left(A^{\mathrm{c}}\right)_{\delta}$ and $\left.c_{k l}\right|_{A_{\delta}} \in W^{2 ; \infty}\left(A_{\delta}\right)$. Then $C_{c}^{\infty}\left(\mathbb{R}^{d}\right)$ is a core of $H$.

Proof. There exists $\chi_{1} \in W^{2, \infty}\left(\mathbb{R}^{d}\right)$ such that $\left.\chi_{1}\right|_{A_{\delta / 2}}=\mathbb{1}$ and $\operatorname{supp} \chi_{1} \subseteq A_{\delta}$. Define $c_{k l}^{(1)}=\chi_{1} c_{k l} \in W^{2, \infty}\left(\mathbb{R}^{d}\right)$. Then $\left.c_{k l}^{(1)}\right|_{A_{\delta / 2}}=\left.c_{k l}\right|_{A_{\delta / 2}}$.

In addition there exists $\chi_{2} \in W^{1, \infty}\left(\mathbb{R}^{d}\right)$ such that $\left.\chi_{2}\right|_{\left(A^{\mathrm{c}}\right)_{\delta / 2}}=\mathbb{1}$ and $\operatorname{supp} \chi_{2} \subseteq\left(A^{\mathrm{c}}\right)_{\delta}$. Define $c_{k l}^{(2)}=\chi_{2} c_{k l}+\left(\mathbb{1}-\chi_{2}\right) \delta_{k l} \in W^{1, \infty}\left(\mathbb{R}^{d}\right)$.

Let $H^{(1)}$ and $H^{(2)}$ be the degenerate elliptic operator with coefficients $\left(c_{k l}^{(1)}\right)$ and $\left(c_{k l}^{(2)}\right)$. Then the statement of the theorem follows by applying Proposition 4.1 (II) to $H^{(1)}$, Proposition 4.1(I) to $H^{(2)}$ and using Proposition 4.5.

The condition that $C_{c}^{\infty}\left(\mathbb{R}^{d}\right)$ is a core of $H$ does not follow in general from the assumption that the coefficients are in $W^{1, \infty}\left(\mathbb{R}^{d}\right)$. The situation is clarified by the onedimensional example considered in [11, Section 5].

Example 4.7. Let $H_{0}=-d c d$ with $D\left(H_{0}\right)=C_{c}^{\infty}(\mathbb{R})$ and with $c(x)=\left(x^{2} /\left(1+x^{2}\right)\right)^{\gamma / 2}$ where $\gamma \geq 1$. Thus $c \in W^{\gamma, \infty}(\mathbb{R})$. It follows by the arguments in [4, Proposition 3.5] (see also [26, Theorem 1.1]) that $C_{c}^{\infty}(\mathbb{R})$ is a core of $H_{p}$ if and only if $\gamma \geq 2-1 / p$. In particular if $\gamma=1$, which corresponds to the $W^{1, \infty}$ case, then $C_{c}^{\infty}(\mathbb{R})$ is a core of $H_{1}$ but is not a core of $H_{p}$ for any $p>1$. If, however, $\gamma \geq 2$, the $W^{2, \infty}$ case, then $C_{c}^{\infty}(\mathbb{R})$ is a core of $H_{p}$ for all $p \in[1, \infty]$. Finally, note that $C_{c}^{\infty}(\mathbb{R})$ is a core of $H_{2}$ if and only if $\gamma \geq 3 / 2$.

Theorem 4.6 establishes that double differentiability in a $\delta$-neighbourhood of the set of $x \in \mathbb{R}^{d}$ for which $C(x)$ is not invertible suffices for $C_{c}^{\infty}\left(\mathbb{R}^{d}\right)$ to be a core of $H$. In one dimension this is precisely the set

$$
\mathcal{Z}=\left\{x \in \mathbb{R}^{d}: C(x)=0\right\}
$$

where $C$ vanishes, and in higher dimensions it corresponds to the set of $x$ for which $\mu_{C}(x)=0$. The differentiability condition can, however, be weakened around the boundary of $\mathcal{Z}$. If there exists $\delta>0$ such that $c_{k l} \in W^{2, \infty}\left(\mathcal{Z}_{\delta}\right)$ for all $k, l \in\{1, \ldots, d\}$ then there is $a>0$ such that $C(x) \leq a d_{\mathcal{Z}}(x)^{2} I$ for all $x \in \mathcal{Z}_{\delta / 2}$. This follows since the matrix $C$ is positive semidefinite. In Corollary 4.12 we establish that the core property follows with this weaker degeneracy condition replacing the $W^{2, \infty}$ condition under the assumption that for every $x \in \mathbb{R}^{d}$ the matrix $C(x)$ is either invertible or zero. The corollary is a consequence of the subsequent analysis of core properties for $p \in[1,2]$.

Our analysis of core properties is based on an $L_{p}$ extension of an argument used to prove essential self-adjointness of Schrödinger operators and elliptic operators on manifolds by various authors. A clear explanation of the $L_{2}$ estimates is given in [5, Section 3] together with references to earlier works. Moreover, [27, Proposition 6.1] gives a straightforward $L_{p}$ extension, valid for $p \in[1,2]$, of the principal $L_{2}$ estimate [5, Theorem 3.1]. (See also [29].) But for current applications a more sophisticated localized extension is required.

Throughout the rest of this paper, we denote by $\mathcal{Z}$ the set (4.3) of all $x \in \mathbb{R}^{d}$ for which $C(x)=0$. Since the coefficients $c_{k l}$ are continuous, the set $\mathcal{Z}$ is closed and its 
complement $\mathcal{Z}^{\mathrm{c}}$ is open. Further, if $p \in[1,2]$, then we define $r=p /(2-p) \in[1, \infty]$, with the convention that $r=\infty$ if $p=2$.

Theorem 4.8. Let $p \in[1,2]$. Assume that $|\partial \mathcal{Z}|=0$ and $C(x)$ is invertible for each $x \in \mathcal{Z}^{\mathrm{c}}$. Further, assume that, for every bounded measurable set $D \subset \mathbb{R}^{d}$, there exist $\eta_{1}, \eta_{2}, \ldots \in W^{1, \infty}\left(\mathbb{R}^{d}\right)$ such that $\operatorname{supp} \eta_{n} \subseteq \mathcal{Z}^{\mathrm{c}}$ and $0 \leq \eta_{n} \leq \mathbb{1}$ for all $n \in \mathbb{N}$,

$$
\lim _{n \rightarrow \infty} \int_{D}\left|\eta_{n} \varphi\right|^{2}=\int_{D}\left|\mathbb{1}_{\mathcal{Z}^{c}} \varphi\right|^{2}
$$

for all $\varphi \in L_{2}(D)$ and

$$
\lim _{n \rightarrow \infty}\left\|\mathbb{1}_{D} \Gamma\left(\eta_{n}\right)\right\|_{p /(2-p)}=0,
$$

where $\Gamma$ is given by (4.1). Then $C_{c}^{\infty}\left(\mathbb{R}^{d}\right)$ is a core of $H_{p}$.

Proof. It suffices to prove that $\left(\lambda I+H_{p}\right) C_{c}^{\infty}\left(\mathbb{R}^{d}\right)$ is dense in $L_{p}\left(\mathbb{R}^{d}\right)$ for a sufficiently large $\lambda>0$.

Fix $\tau \in C_{c}^{\infty}\left(\mathbb{R}^{d}\right)$ such that $0 \leq \tau \leq \mathbb{1}$, supp $\tau \subset B_{2}(0)$ and $\tau(x)=1$ for all $x \in B_{1}(0)$. There exists $\lambda_{0}>0$ such that $|(\nabla \tau)(x)| \leq \lambda_{0}$ for all $x \in \mathbb{R}^{d}$. Define $\lambda=4^{d+1} \lambda_{0}\|C\|_{\infty}$, where $\|C\|_{\infty}=\sup \left\{\|C(x)\|: x \in \mathbb{R}^{d}\right\}$.

Suppose that $\varphi \in L_{q}\left(\mathbb{R}^{d}\right)$, where $q$ is the dual exponent of $p$, and suppose also that $\left(\varphi,\left(\lambda I+H_{p}\right) \psi\right)=0$ for all $\psi \in C_{c}^{\infty}\left(\mathbb{R}^{d}\right)$. Then $(\varphi, \psi)=0$ for all $\psi \in C_{c}^{\infty}\left(\mathcal{Z}^{\circ}\right)$ and $\left.\varphi\right|_{Z^{\circ}}=0$. Since $|\partial \mathcal{Z}|=0$, one also has $\left.\varphi\right|_{\partial \mathcal{Z}}=0$. So it remains to show that $\left.\varphi\right|_{Z^{c}}=0$.

Note that $\varphi \in L_{2, \text { loc }}\left(\mathbb{R}^{d}\right)$, as $q \geq 2$. Since $(\varphi,(\lambda I+H) \psi)=0$ for all $\psi \in C_{c}^{\infty}\left(\mathcal{Z}^{\mathrm{c}}\right)$, it follows from local ellipticity that $\varphi \in W_{\text {loc }}^{1,2}\left(\mathcal{Z}^{\mathrm{c}}\right)$. Let $\rho \in C_{c}^{\infty}\left(\mathbb{R}^{d}\right)$ such that $0 \leq \rho \leq \mathbb{1}$ and $\rho \neq 0$. Write $D=\operatorname{supp} \rho$. By assumption, there exist $\eta_{1}, \eta_{2}, \ldots \in W^{1, \infty}\left(\mathbb{R}^{d}\right)$ such that supp $\eta_{n} \subseteq \mathcal{Z}^{\mathrm{c}}$ and $0 \leq \eta_{n} \leq \mathbb{1}$ for all $n \in \mathbb{N}, \lim \int_{D}\left|\eta_{n} \psi\right|^{2}=\int_{D}\left|\mathbb{1}_{\mathcal{Z}^{\mathrm{c}}} \psi\right|^{2}$ for all $\psi \in L_{2}(D)$, and $\lim \left\|\mathbb{1}_{D} \Gamma\left(\eta_{n}\right)\right\|_{r}=0$.

First, if $\tau_{1}, \tau_{2} \in C_{c}^{\infty}\left(\mathcal{Z}^{\mathrm{c}}\right)$ with $\left.\tau_{2}\right|_{\operatorname{supp} \tau_{1}}=\mathbb{1}$, then

$$
\left(\tau_{2} \varphi,(\lambda I+H)\left(\tau_{1} \psi\right)\right)=\left(\varphi,(\lambda I+H)\left(\tau_{1} \psi\right)\right)=0
$$

for all $\psi \in C_{c}^{\infty}\left(\mathbb{R}^{d}\right)$. Hence

$$
\lambda\left(\varphi, \tau_{1} \psi\right)+h\left(\tau_{2} \varphi, \tau_{1} \psi\right)=0
$$

for all $\psi \in C_{c}^{\infty}\left(\mathbb{R}^{d}\right)$, and then by density for all $\psi \in W^{1,2}\left(\mathbb{R}^{d}\right)$. Second, for all $n \in \mathbb{N}$, set $\tilde{\eta}_{n}=\rho \eta_{n}$. Then $\tilde{\eta}_{n} \in W^{1, \infty}\left(\mathbb{R}^{d}\right)$ with compact support in $\mathcal{Z}^{\mathrm{c}}$. Now choose $\tau_{1}$ such that $\left.\tau_{1}\right|_{\text {supp } \tilde{\eta}_{n}}=\mathbb{1}$ and set $\psi=\tilde{\eta}_{n}^{2} \varphi$. It follows that $\psi \in W^{1,2}\left(\mathbb{R}^{d}\right)$ and $\tau_{1} \psi=\psi$. Therefore one may estimate that

$$
\begin{aligned}
\lambda\left\|\tilde{\eta}_{n} \varphi\right\|_{2}^{2} & =-h\left(\tau_{2} \varphi, \tilde{\eta}_{n}^{2} \varphi\right) \\
& =-\sum_{k, l=1}^{d} \int c_{k l}\left(\partial_{k}\left(\tilde{\eta}_{n} \varphi\right)\right)\left(\partial_{l}\left(\tilde{\eta}_{n} \varphi\right)\right)+\sum_{k, l=1}^{d} \int c_{k l} \varphi^{2}\left(\partial_{k} \tilde{\eta}_{n}\right)\left(\partial_{l} \tilde{\eta}_{n}\right)
\end{aligned}
$$




$$
\begin{aligned}
& \leq \int \varphi^{2} \Gamma\left(\rho \eta_{n}\right) \\
& \leq 2 \int \varphi^{2} \rho^{2} \Gamma\left(\eta_{n}\right)+2 \int \varphi^{2} \Gamma(\rho) \eta_{n}^{2} \\
& \leq 2\|\varphi\|_{q}^{2}\left\|\rho^{2} \Gamma\left(\eta_{n}\right)\right\|_{r}+2 \int \varphi^{2} \Gamma(\rho) \eta_{n}^{2},
\end{aligned}
$$

where the last step uses the Hölder inequality. So the limit $n \rightarrow \infty$ gives

$$
\lambda \int \rho^{2} \varphi^{2} \mathbb{1}_{\mathcal{Z}^{\mathrm{c}}} \leq 2 \int \Gamma(\rho) \varphi^{2} \mathbb{1}_{\mathcal{Z}^{\mathrm{c}}}
$$

and this is valid for all $\rho \in C_{c}^{\infty}\left(\mathbb{R}^{d}\right)$.

Next define $\tau_{n} \in C_{c}^{\infty}\left(\mathbb{R}^{d}\right)$ by $\tau_{n}(x)=\tau\left(2^{-n} x\right)$ for each $n \in \mathbb{N}$, and then define $a_{n}=$ $\int \tau_{n}^{2} \varphi^{2} \mathbb{1}_{\mathcal{Z}^{\mathfrak{c}}}$. Then, since $r \geq 1$ and $\left|B_{1}(0)\right| \geq 1$, one has

$$
a_{n} \leq\|\varphi\|_{q}^{2}\left\|\tau_{n}^{2}\right\|_{r} \leq\|\varphi\|_{q}^{2}\left|B_{2^{n+1}}(0)\right|^{1 / r} \leq\|\varphi\|_{q}^{2}\left|B_{1}(0)\right| 2^{(n+1) d} .
$$

So the series $\sum 4^{-n d} a_{n}$ converges. But $\left|\nabla \tau_{n}\right| \leq 2^{-n} \lambda_{0} \tau_{n+1}$ for all $n \in \mathbb{N}$, by the definition of $\lambda_{0}$. Hence it follows from (4.5), with $\rho$ replaced by $\tau_{n}$, that

$$
\begin{aligned}
\lambda \sum_{n=1}^{\infty} 4^{-n d} a_{n} & \leq 2 \sum_{n=1}^{\infty} 4^{-n d} \int \varphi^{2} \mathbb{1}_{\mathcal{Z}^{\mathrm{c}}\|C\|_{\infty}\left(2^{-n} \lambda_{0} \tau_{n+1}\right)^{2}} \\
& \leq \lambda_{0}^{2}\|C\|_{\infty} \sum_{n=1}^{\infty} 4^{-n d} a_{n+1} \\
& \leq 4^{d} \lambda_{0}^{2}\|C\|_{\infty} \sum_{n=1}^{\infty} 4^{-n d} a_{n}=4^{-1} \lambda \sum_{n=1}^{\infty} 4^{-n d} a_{n} .
\end{aligned}
$$

Therefore $\sum_{n=1}^{\infty} 4^{-n d} a_{n}=0$ and $a_{n}=0$ for all $n \in \mathbb{N}$. So $\varphi \mathbb{1}_{\mathcal{Z}^{\mathrm{c}} \cap B_{2^{n}}(0)}=0$ for all $n \in \mathbb{N}$ and $\varphi \mathbb{1}_{\mathcal{Z}^{\mathrm{c}}}=0$. Thus $\varphi=0$.

A similar result is valid for open sets $\Omega$ instead of $\mathbb{R}^{d}$. This is particularly interesting for applications to $\mathcal{Z}^{\mathrm{c}}$ and connected components of $\mathcal{Z}^{\mathrm{c}}$.

Remark 4.9. Suppose that $p \in[1,2]$ and that $\Omega \subset \mathbb{R}^{d}$ is open. Assume that $C(x)$ is invertible for each $x \in \Omega$ and that for every bounded measurable subset $D$ of $\Omega$ there exist $\eta_{1}, \eta_{2}, \ldots \in W^{1, \infty}(\Omega)$ such that $\operatorname{supp} \eta_{n} \subseteq \Omega$ and $0 \leq \eta_{n} \leq \mathbb{1}$ for all $n \in \mathbb{N}$, $\lim \int_{D}\left|\eta_{n} \varphi\right|^{2}=\int_{D}|\varphi|^{2}$ for all $\varphi \in L_{2}(D)$ and

$$
\lim _{n \rightarrow \infty}\left\|\mathbb{1}_{D} \Gamma^{\Omega}\left(\eta_{n}\right)\right\|_{p /(2-p)}=0
$$

where $\Gamma^{\Omega}(\rho)=\sum c_{k l}\left(\partial_{k} \rho\right)\left(\partial_{l} \rho\right) \in L_{\infty}(\Omega)$. Define $h_{0, \Omega}: C_{c}^{\infty}(\Omega) \rightarrow \mathbb{R}$ by

$$
h_{0, \Omega}(\varphi)=\sum_{k, l=1}^{d} \int_{\Omega} c_{k l}\left(\partial_{k} \varphi\right)\left(\partial_{l} \varphi\right) \text {. }
$$


The form $h_{0, \Omega}$ is closable and its closure is a Dirichlet form in $L_{2}(\Omega)$. Let $H_{\Omega, p}$ be the associated semigroup generator in $L_{p}(\Omega)$. Then $C_{c}^{\infty}(\Omega)$ is a core of $H_{\Omega, p}$.

The conclusion follows by a modification of the proof of Theorem 4.8. Now $\varphi \in L_{q}(\Omega)$ and one replaces $\mathcal{Z}^{\mathrm{c}}$ by $\Omega$. One still chooses $\rho \in C_{c}^{\infty}\left(\mathbb{R}^{d}\right)$ but now $D=\Omega \cap \operatorname{supp} \rho$. Then $\tilde{\eta}_{n}=\rho \eta_{n}$ has compact support in $\Omega$ and one deduces as before that

$$
\lambda \int_{\Omega} \rho^{2} \varphi^{2} \leq 2 \int_{\Omega} \varphi^{2} \Gamma(\rho)
$$

for all $\rho \in C_{c}^{\infty}\left(\mathbb{R}^{d}\right)$. This is a new version of (4.5). The subsequent argument is a repetition of the proof of Theorem 4.8 .

The next result is essentially a corollary of Theorem 4.8 combined with the capacity estimates of [27, Proposition 4.3]. It exploits two distinct features of the degenerate operator $H_{0}$. The first feature is the order of degeneracy of the coefficients $c_{k l}$ near the boundary $\partial \mathcal{Z}$. The second is the effective dimension of this boundary. Recall that the Minkowski dimension of a bounded nonempty subset $A$ of $\mathbb{R}^{d}$ is defined by

$$
d_{\text {Min }}=d-\lim _{\delta \rightarrow 0_{+}} \frac{\log \left|A_{\delta}\right|}{\log \delta}
$$

whenever the limit exists. The next theorem introduces local versions of the order of degeneracy and the boundary dimension. By this we mean that the values of the parameters $\gamma$ and $d_{m}$ occurring in the following statement may depend on $D$.

Theorem 4.10. Let $p \in[1,2]$. Assume that $\mathcal{Z} \neq \emptyset,|\partial \mathcal{Z}|=0$ and $C(x)$ is invertible for each $x \in \mathcal{Z}^{\mathrm{c}}$. Further, assume that for every nonempty bounded set $D \subset \mathbb{R}^{d}$ there are $a, b>0, d_{m} \in[0, d]$ and $\gamma \in[1,2]$ such that

$$
\begin{gathered}
(2-\gamma) p \leq\left(d-d_{m}\right)(2-p), \\
C(x) \leq a d_{\mathcal{Z}}(x)^{\gamma} I \quad \text { and } \quad\left|\left(\mathcal{Z} \cap D_{1}\right)_{\delta}\right| \leq b \delta^{d-d_{m}}
\end{gathered}
$$

for all $x \in D$ and $\delta \in(0,1]$. Then $C_{c}^{\infty}\left(\mathbb{R}^{d}\right)$ is a core of $H_{p}$.

Proof. For all $n \in \mathbb{N}$, set $\eta_{n}=\chi_{n} \circ d_{\mathcal{Z}}$, where $\chi_{n}$ is given by (3.6). Then $\eta_{n} \in W^{1, \infty}\left(\mathbb{R}^{d}\right)$, $\operatorname{supp} \eta_{n} \subset \mathcal{Z}^{\mathrm{c}}$ and $0 \leq \eta_{n} \leq \mathbb{1}$. Moreover, $\lim \eta_{n}=\mathbb{1}_{\mathcal{Z}^{\mathrm{c}}}$ pointwise.

Let $D \subset \mathbb{R}^{d}$ be bounded. We may assume that $D \neq \emptyset$. By assumption there exist $a, b>0, d_{m} \in[0, d]$ and $\gamma \in[1,2]$ such that $(2-\gamma) r \leq\left(d-d_{m}\right), C(x) \leq a d_{\mathcal{Z}}(x)^{\gamma} I$ for all $x \in D$ and $\left|\left(\mathcal{Z} \cap D_{1}\right)_{\delta}\right| \leq b \delta^{d-d_{m}}$ for all $\delta \in(0,1]$. Clearly $\lim \int_{D}\left|\eta_{n} \varphi\right|^{2}=\int_{D}\left|\mathbb{1}_{\mathcal{Z}^{c}} \varphi\right|^{2}$ for all $\varphi \in L_{2}(D)$.

If $p=2$, then $r=\infty$ and $\gamma=2$. But

$$
\left\|\mathbb{1}_{D} \Gamma\left(\chi_{n} \circ d_{\mathcal{Z}}\right)\right\|_{\infty} \leq\|C\|_{\infty}(\log n)^{-2} .
$$


Therefore (4.4) is valid. Alternatively, suppose that $p<2$. Then $r<\infty$ and

$$
\begin{aligned}
\left\|\mathbb{1}_{D} \Gamma\left(\chi_{n} \circ d_{\mathcal{Z}}\right)\right\|_{r}^{r} & \leq a^{r}(\log n)^{-2 r} \int_{\left[n^{-1} \leq d_{\mathcal{Z}} \leq 1\right] \cap D} d_{\mathcal{Z}}^{-(2-\gamma) r} \\
& =a^{r}(\log n)^{-2 r} \int_{\left[n^{-1} \leq d_{\mathcal{Z}} \leq 1\right] \cap D} d x\left(1+(2-\gamma) r \int_{d_{\mathcal{Z}}(x)}^{1} d \delta \delta^{-1-(2-\gamma) r}\right) \\
& =a^{r}(\log n)^{-2 r}\left(|D|+(2-\gamma) r \int_{1 / n}^{1} d \delta \delta^{-1-(2-\gamma) r}\left|\mathcal{Z}_{\delta} \cap D\right|\right) .
\end{aligned}
$$

But $\mathcal{Z}_{\delta} \cap D \subseteq\left(\mathcal{Z} \cap D_{1}\right)_{\delta}$ for all $\delta \in(0,1]$. Hence $\left|\mathcal{Z}_{\delta} \cap D\right| \leq b \delta^{d-d_{m}}$ for all $\delta \in(0,1]$. Therefore

$$
\begin{aligned}
\int_{1 / n}^{1} d \delta \delta^{-1-(2-\gamma) r}\left|\mathcal{Z}_{\delta} \cap D\right| & \leq b \int_{1 / n}^{1} d \delta \delta^{-1-(2-\gamma) r+\left(d-d_{m}\right)} \\
& \leq b \int_{1 / n}^{1} d \delta \delta^{-1}=b \log n,
\end{aligned}
$$

since $\left(d-d_{m}\right)-(2-\gamma) r \geq 0$. Therefore (4.4) is again valid and one can apply Theorem 4.8 to deduce the desired result.

REMARK 4.11. If one applies Theorem 4.10 to the operator considered in Example 4.7, then $d=1, \mathcal{Z}=\{0\}$ and $d_{m}=0$. Therefore one concludes that $C_{c}^{\infty}(\mathbb{R})$ is a core of $H_{p}$ if $(2-\gamma) p \leq(2-p)$ or, equivalently, if $\gamma \geq 3-2 / p$. But the exact result is $\gamma \geq 2-1 / p$. One concludes that the estimation method used to establish the theorem is not optimal unless $p=1$.

Theorem 4.10 has two straightforward corollaries.

Corollary 4.12. Assume that $\mathcal{Z} \neq \emptyset,|\partial \mathcal{Z}|=0$ and $C(x)$ is invertible for all $x \in \mathcal{Z}^{\mathrm{c}}$. Further, assume that for every bounded subset $D \subset \mathbb{R}^{d}$ there exists $a>0$ such that

$$
C(x) \leq a d_{Z}(x)^{2} I
$$

for all $x \in D$. Then $C_{c}^{\infty}\left(\mathbb{R}^{d}\right)$ is a core for $H\left(=H_{2}\right)$.

Proof. Apply Theorem 4.10 with $p=2, \gamma=2$ and $d_{m}=d$.

Corollary 4.13. Assume that $\mathcal{Z} \neq \emptyset,|\partial \mathcal{Z}|=0$ and $C(x)$ is invertible for all $x \in \mathcal{Z}^{\mathrm{c}}$. Further, assume that $\mathcal{Z}^{\mathrm{c}}$ has a locally Lipschitz boundary. Then $C_{c}^{\infty}\left(\mathbb{R}^{d}\right)$ is a core for $H_{1}$.

Proof. Apply Theorem 4.10 with $p=1, \gamma=1$ and $d_{m}=d-1$. (The necessary bounds $C(x) \leq a d_{\mathcal{Z}}(x) I$ follow because $c_{k l} \in W^{1, \infty}\left(\mathbb{R}^{d}\right)$.)

Finally, we consider the situation for which the Minkowski dimension of the boundary exists. Note that even if the Minkowski dimension of the bounded nonempty set $A$ exists, this does not imply that there exists $b>0$ such that

$$
\left|A_{\delta}\right| \leq b \delta^{d-d_{\text {Min }}}
$$


for all $\delta \in(0,1]$. Therefore the degeneracy criterion (4.6) is in general only valid if $d_{m}>d_{\text {Min }}$. Nevertheless there are some interesting fractal cases for which the conclusions of Theorem 4.10 persist with $d_{m}=d_{\text {Min }}$.

The Minkowski dimension always exists for self-similar fractals and is equal to the Hausdorff dimension. But the bound (4.8) does not necessarily exist uniformly for all $\delta \in(0,1]$. If, however, the contractions defining the fractal satisfy the open set condition (see [17, Definition 5.2(1)]) then the Minkowski dimension can be used in (4.6).

Theorem 4.14. Let $p \in[1,2]$. Further, let $\Omega$ be a bounded open set whose boundary $A=\partial \Omega$ is a self-similar fractal for which the defining contractions satisfy the open set condition. Let $d_{\text {Min }}$ denote the Minkowski dimension of $A$.

Assume that $|A|=0$ and $C(x)$ is invertible for each $x \in A^{\mathrm{c}}$. Further, assume that there exist $\gamma \in[1,2]$ and $a>0$ such that

$$
(2-\gamma) p \leq\left(d-d_{\operatorname{Min}}\right)(2-p)
$$

and $C(x) \leq a d_{A}(x)^{\gamma} I$ for all $x \in \mathbb{R}^{d}$. Then $C_{c}^{\infty}\left(\mathbb{R}^{d}\right)$ is a core of $H_{p}$.

Proof. The proof is a variation of the proof of Theorem 4.10, and we adopt the same notation. If $p=2$ then $\gamma=2$, and one can argue as before.

Suppose that $p \neq 2$. It follows from [15, Theorem 2.3(ii)] that the mean

$$
L=\lim _{T \rightarrow \infty} T^{-1} \int_{0}^{T} d t \frac{\left|A_{e^{-t}}\right|}{e^{-t\left(d-d_{\text {Min }}\right)}}
$$

exists. Then for all large $n \in \mathbb{N}$ one estimates

$$
\begin{aligned}
\int_{1 / n}^{1} d \delta \delta^{-1-(2-\gamma) r}\left|A_{\delta}\right| & \leq \int_{1 / n}^{1} d \delta \delta^{-1-\left(d-d_{\text {Min }}\right)}\left|A_{\delta}\right| \\
& =\int_{0}^{\log n} d t \frac{\left|A_{e^{-t}}\right|}{e^{-t\left(d-d_{\text {Min }}\right)}} \leq(L+1) \log n .
\end{aligned}
$$

This is the new version of (4.7). The remainder of the proof is evident.

\section{Illustrative examples}

Theorem 1.1 provides a practical criterion for invariance of a subspace $L_{2}(\Omega)$ under the diffusion semigroup $S$; it suffices to prove invariance under the flows $T^{(1)}, \ldots, T^{(d)}$. The latter property is, however, simpler to establish since the action of the $T^{(k)}$ is straightforward. We illustrate this with four examples.

Example 5.1 (One dimension). If $d=1$, then the elliptic operator $H$ is given by one nonnegative coefficient $c \in W^{1, \infty}(\mathbb{R})$ and $H_{0} \varphi=-\left(c \varphi^{\prime}\right)^{\prime}$ for all $\varphi \in C_{c}^{\infty}(\mathbb{R})$. Now $\mathcal{Z}=\{x \in \mathbb{R}: c(x)=0\}$ is the zero set of the coefficient $c$. Let $a \in \mathcal{Z}$. We argue that $L_{2}(a, \infty)$ is invariant under $S$.

Since $c \in W^{1, \infty}(\mathbb{R})$, the zero at the point $a$ must be of order at least one. In particular, $c^{-1}$ is not integrable at $a$. Now $\left(T_{t} \varphi\right)(x)=\varphi\left(\omega_{t}(x)\right)$, where $t \mapsto \omega_{t}(x)$ is the unique 
solution of the differential equation $(d / d t) \omega_{t}(x)=c\left(\omega_{t}(x)\right)$ with initial value $\omega_{0}(x)=x$. Therefore it follows from the standard theory of differential equations that $L_{2}(a, \infty)$ is $T$-invariant. But $(a, \infty)$ has a Lipschitz boundary. Then by Theorem 1.1 the subspace $L_{2}(a, \infty)$ is $S$-invariant. Hence $L_{2}(-\infty, a)$ is also $S$-invariant.

In addition, if $a, b \in \mathcal{Z}$ with $a<b$, then

$$
L_{2}(a, b)=L_{2}(-\infty, b) \cap L_{2}(a, \infty)
$$

is $S$-invariant. One concludes that if $\left\{I_{\alpha}: \alpha \in A\right\}$ are the connected components of $\mathcal{Z}^{\mathrm{c}}$ with $I_{\alpha} \neq I_{\beta}$ if $\alpha \neq \beta$, then $S_{t}=\bigoplus_{\alpha} S_{t}^{(\alpha)}$ for all $t>0$, where $S^{(\alpha)}$ is the restriction of $S$ to $L_{2}\left(I_{\alpha}\right)$. Let $\alpha \in A$. Then it follows from Remark 4.9 that $C_{c}^{\infty}\left(I_{\alpha}\right)$ is a core for the operator $H_{I_{\alpha}, 1}$, where we use the notation of the remark. But the generator of $S^{(\alpha)(1)}$ (on $L_{1}\left(I_{\alpha}\right)$ ) is an extension of the operator $\left.H_{I_{\alpha}, 1}\right|_{C_{c}^{\infty}\left(I_{\alpha}\right)}$. Hence $H_{I_{\alpha}, 1}$ is the generator of the semigroup $S^{(\alpha)(1)}$. Then $H_{I_{\alpha}}$ is the generator of $S^{(\alpha)}$.

Note that since $c>0$ on each of the connected components $I_{\alpha}$ of $\mathcal{Z}^{\mathrm{c}}$ it follows that each $S^{(\alpha)}$ is irreducible by [27, Proposition 6.10]. (Here we use the terminology of [21, Definition 2.8].) In particular, the $S^{(\alpha)}$ are ergodic, that is, there are no nontrivial closed $S^{(\alpha)}$-invariant subspaces of $L_{2}\left(I_{\alpha}\right)$ (see [21, Theorem 2.9]).

Theorem 1.1 also allows one to establish invariance properties for the semigroup associated with an elliptic operator with bounded measurable coefficients. These operators are introduced by a variation of the definitions of Section 1.

First, if $C=\left(c_{k l}\right)$ is a positive semidefinite matrix over $\mathbb{R}^{d}$ with real coefficients $c_{k l}=$ $c_{l k} \in L_{\infty}\left(\mathbb{R}^{d}\right)$, then $\varphi \mapsto \sum_{k, l=1}^{d}\left(\partial_{k} \varphi, c_{k l} \partial_{l} \varphi\right)$ with domain $C_{c}^{\infty}\left(\mathbb{R}^{d}\right)$ is a positive, densely defined, quadratic form on $L_{2}\left(\mathbb{R}^{d}\right)$. But it is not necessarily closable. Nevertheless one can define its relaxation, or viscosity closure, $h$ (see, for example, [11] and references therein). Then $h$ is a Dirichlet form and the corresponding self-adjoint operator $H$ generates a sub-Markovian semigroup $S$. If the coefficients $\left(c_{k l}\right)$ are Lipschitz then this definition, which we now adopt, coincides with the definition of Section 1. (For a direct definition of the operator $H$ see [2, Theorem 1.1].)

Invariance for operators with measurable coefficients can be deduced from invariance for operators with Lipschitz coefficients by the following comparison result.

Lemma 5.2. Let $H^{(1)}$ and $H^{(2)}$ be two degenerate elliptic operators with bounded real symmetric measurable coefficients $C^{(1)}$ and $C^{(2)}$, and $S^{(1)}$ and $S^{(2)}$ be the corresponding semigroups. Further, let $\Omega$ be a measurable subset of $\mathbb{R}^{d}$.

If $C^{(2)} \leq C^{(1)}$ and $L_{2}(\Omega)$ is $S^{(1)}$-invariant, then $L_{2}(\Omega)$ is also $S^{(2)}$-invariant.

Proof. This follows from [8, Corollary 2.11].

ExAmple 5.3 (Lipschitz domain). Let $H$ be the elliptic operator on $L_{2}\left(\mathbb{R}^{d}\right)$ with bounded real symmetric measurable coefficients $C=\left(c_{k l}\right)$. Let $\Omega \subseteq \mathbb{R}^{d}$ be open with locally Lipschitz boundary. Assume that $A=\partial \Omega \neq \emptyset$ and there is $a>0$ such that $C \leq a d_{A} I$. Then $L_{2}(\Omega)$ is invariant under the semigroup $S$ generated by $H$. This is established as follows. 
Since the coefficients are bounded, there exists $M>0$ such that $C \leq M I$. Set $c=a d_{A} \wedge M$. Then $c \in W^{1, \infty}\left(\mathbb{R}^{d}\right)$ and $C \leq c I$. Let $H_{A}$ denote the elliptic operator with coefficient matrix $c I$. Since $c \in W^{1, \infty}\left(\mathbb{R}^{d}\right)$, it follows from Theorem 1.1, Proposition 1.2 and Lemma 5.2 that it suffices to prove that $L_{2}(\Omega)$ is invariant under the flows $T^{\psi}$ with coefficients $y_{\psi, k}=c\left(\partial_{k} \psi\right)$ for all $\psi \in C_{c}^{\infty}\left(\mathbb{R}^{d}\right)$.

Because $d_{A}(x)=0$ for all $x \in A$, it follows that $c(x)=0$ and $y_{\psi, k}(x)=0$ for all $x \in A$ and $k \in\{1, \ldots, d\}$. Therefore $e^{t Y_{\psi}} x=x$ for all $x \in A$ and $t \in \mathbb{R}$. Let $y \in \Omega$. Suppose that there exists $t \in \mathbb{R}$ such that $e^{t Y_{\psi}} y \notin \Omega$. We may assume that $t>0$. Since the orbit $s \mapsto e^{s Y_{\psi}} y$ is continuous, there must be an intermediate value $u \in(0, t]$ for which $e^{u Y_{\psi}} y \in A$. But then

$$
e^{t Y_{\psi}} y=e^{(t-u) Y_{\psi}}\left(e^{u Y_{\psi}} y\right)=e^{u Y_{\psi}} y \in A,
$$

which is a contradiction. Therefore $e^{t Y_{\psi}} \Omega \subseteq \Omega$ and $T_{t}^{\psi} L_{2}(\Omega) \subseteq L_{2}(\Omega)$ for all $t \in \mathbb{R}$.

It is possible to characterize the generator of the restriction of $S$ to $L_{2}(\Omega)$. If $\chi_{n}$ is as in (3.6) and $\eta_{n}=\left.\left(\chi_{n} \circ d_{A}\right)\right|_{\Omega}$, then

$$
\lim _{n \rightarrow \infty}\left\|\mathbb{1}_{D} \sum_{k=1}^{d} c\left|\partial_{k} \eta_{n}\right|^{2}\right\|_{L_{1}(\Omega)}=0
$$

for every bounded measurable set $D \subset \mathbb{R}^{d}$, by the arguments to prove Corollary 4.13. Then, by the domination assumption, one also has

$$
\lim _{n \rightarrow \infty}\left\|\mathbb{1}_{D} \sum_{k, l=1}^{d} c_{k l}\left(\partial_{k} \eta_{n}\right)\left(\partial_{l} \eta_{n}\right)\right\|_{L_{1}(\Omega)}=0 .
$$

It follows by Remark 4.9 that $C_{c}^{\infty}\left(\mathbb{R}^{d}\right)$ is a core for the operator $H_{\Omega, 1}$, where we use the notation of the remark. Then it follows, as in Example 5.1, that $H_{\Omega}$ is the generator of the restriction of $S$ to $L_{2}(\Omega)$.

Example 5.3 is based on the second invariance criterion of Theorem 1.1, the Lipschitz property of the open set. But one can also derive an analogous statement from the first criterion of the theorem, the core property of $C_{c}^{\infty}\left(\mathbb{R}^{d}\right)$. In particular, Theorem 4.10 establishes this latter property from a second-order degeneracy on the boundary.

EXAmple 5.4 (General domain). Let $H$ be the degenerate elliptic operator with bounded real symmetric measurable coefficients $C=\left(c_{k l}\right)$. Let $\Omega \subseteq \mathbb{R}^{d}$ be open with boundary $A=\partial \Omega$. Assume that $A \neq \emptyset$ and $|A|=0$. Further, assume that there is $a>0$ such that $C \leq a\left(d_{A}\right)^{2} I$. We again argue that the subspace $L_{2}(\Omega)$ is invariant under the semigroup $S$ generated by $H$.

Let $M>0$ be such that $C \leq M I$. Set $c=a\left(d_{A}\right)^{2} \wedge M$. Let $H_{A}$ denote the elliptic operator with coefficient matrix $c I$. Then $c \in W^{1, \infty}\left(\mathbb{R}^{d}\right), C \leq c I \leq a\left(d_{A}\right)^{2} I$ and $c(x) I$ is invertible for each $x \in A^{\mathrm{c}}$. But now it follows from Corollary 4.12 that $C_{c}^{\infty}\left(\mathbb{R}^{d}\right)$ is a core of $H_{A}$. Hence, by Theorem 1.1, Proposition 1.2 and Lemma 5.2, it suffices to prove that the subspace $L_{2}(\Omega)$ is invariant under the flows $T^{\psi}$ with coefficients $y_{\psi, k}=c\left(\partial_{k} \psi\right)$ for all $\psi \in C_{c}^{\infty}\left(\mathbb{R}^{d}\right)$. But this follows by repetition of the argument in Example 5.3. 
As before, the operator $H_{\Omega}$ is the generator of the restriction of $S$ to $L_{2}(\Omega)$. This follows as in Example 5.3, but this time one has to apply Remark 4.9 with $p=2$.

ExAmple 5.5 (The von Koch snowflake). The snowflake is a self-similar fractal in $\mathbb{R}^{2}$ with Minkowski dimension $d_{\text {Min }}=\log 4 / \log 3$. Let $\Omega$ be the bounded subset of $\mathbb{R}^{2}$ which is bounded by the snowflake. Let $H$ be the degenerate elliptic operator on $L_{2}\left(\mathbb{R}^{d}\right)$ with bounded real symmetric measurable coefficients $C=\left(c_{k l}\right) \geq 0$ and assume that there is $a>0$ such that $C \leq a\left(d_{\partial \Omega}\right)^{d_{\mathrm{Min}}} I$. Then $L_{2}(\Omega)$ is invariant under the semigroup $S$ generated by $H$.

This follows from Theorem 4.14 since the defining contractions for the snowflake satisfy the open set condition. One then argues as in Example 5.3.

Further illustrations of invariances and decompositions are given by tilings. For example, assume that one has a tiling of the plane by tiles whose boundaries are selfsimilar fractals of the type covered by Theorem 4.14. (This can be achieved with two sizes of von Koch snowflake in area ratio 1:3.) Then assume that the coefficient matrix $C$ has a degeneracy on the boundary of order greater than or equal to the Minkowski dimension. It follows that the corresponding sub-Markovian semigroup $S$ leaves each component $I_{\alpha}$ of the tiling invariant and one has a corresponding decomposition $S=\bigoplus S^{(\alpha)}$ of $S$ into irreducible components.

\section{Acknowledgements}

Part of this work was carried out whilst the first named author was visiting the Australian National University with partial support from the Centre for Mathematics and its Applications, and part of the work was carried out whilst the second named author was visiting the University of Auckland with financial support from the Faculty of Science.

\section{References}

[1] W. Arendt, 'Characterization of semigroups on Banach spaces', in: One-parameter Semigroups of Positive Operators, Lecture Notes in Mathematics, 1184 (ed. R. Nagel) (Springer, Berlin, 1986), Ch. A-II.

[2] W. Arendt and A. F. M. ter Elst, 'Sectorial forms and degenerate differential operators', J. Operator Theory (2011), to appear, arXiv:0812.3944.

[3] N. Bouleau and F. Hirsch, Dirichlet Forms and Analysis on Wiener Space, de Gruyter Studies in Mathematics, 14 (Walter de Gruyter, Berlin, 1991).

[4] M. Campiti, G. Metafune and D. Pallara, 'Degenerate self-adjoint evolution equations on the unit interval', Semigroup Forum 57 (1998), 1-36.

[5] E. B. Davies, ' $L^{1}$ properties of second order elliptic operators', Bull. Lond. Math. Soc. 17 (1985), 417-436.

[6] N. Dungey, A. F. M. ter Elst and D. W. Robinson, Analysis on Lie Groups with Polynomial Growth, Progress in Mathematics, 214 (Birkhäuser, Boston, 2003).

[7] A. Eberle, Uniqueness and Non-uniqueness of Semigroups Generated by Singular Diffusion Operators, Lecture Notes in Mathematics, 1718 (Springer, Berlin, 1999).

[8] A. F. M. ter Elst and D. W. Robinson, 'Invariant subspaces of submarkovian semigroups', J. Evol. Equ. 8 (2008), 661-671.

[9] A. F. M. ter Elst and D. W. Robinson, 'Uniform subellipticity', J. Operator Theory 62 (2009), 125-149. 
[10] A. F. M. ter Elst, D. W. Robinson, A. Sikora and Y. Zhu, 'Dirichlet forms and degenerate elliptic operators', in: Partial Differential Equations and Functional Analysis, Operator Theory: Advances and Applications, 168 (eds. E. Koelink et al.) (Birkhäuser, Basel, 2006), pp. 73-95.

[11] A. F. M. ter Elst, D. W. Robinson, A. Sikora and Y. Zhu, 'Second-order operators with degenerate coefficients', Proc. Lond. Math. Soc. 95 (2007), 299-328.

[12] L. C. Evans and R. F. Gariepy, Measure Theory and Fine Properties of Functions, Studies in Advanced Mathematics (CRC Press, Boca Raton, FL, 1992).

[13] K. O. Friedrichs, 'Spektraltheorie halbbeschränkter Operatoren und Anwendung auf die Spektralzerlegung von Differentialoperatoren. I', Math. Anal. 109 (1934), 465-487.

[14] M. Fukushima, Y. Oshima and M. Takeda, Dirichlet Forms and Symmetric Markov Processes, de Gruyter Studies in Mathematics, 19 (Walter de Gruyter, Berlin, 1994).

[15] D. Gatzouras, 'Lacunarity of self-similar and stochastically self-similar sets', Trans. Amer. Math. Soc. 352 (2000), 1953-1983.

[16] E. Hille, Lectures on Ordinary Differential Equations (Addison-Wesley, Reading, MA, 1969).

[17] J. E. Hutchinson, 'Fractals and self similarity', Indiana Univ. Math. J. 30 (1981), 713-747.

[18] D. S. Jerison and A. Sánchez-Calle, 'Estimates for the heat kernel for a sum of squares of vector fields', Indiana Univ. Math. J. 35 (1986), 835-854.

[19] T. Kato, Perturbation Theory for Linear Operators, 2nd edn, Grundlehren der Mathematischen Wissenschaften, 132 (Springer, Berlin, 1980).

[20] O. A. Oleı̌nik and E. V. Radkevič, Second Order Equations with Nonnegative Characteristic Form (American Mathematical Society, Providence, RI, 1973).

[21] E.-M. Ouhabaz, Analysis of Heat Equations on Domains, London Mathematical Society Monographs Series, 31 (Princeton University Press, Princeton, NJ, 2005).

[22] F. Riesz and B. Sz. -Nagy, Leçons d'analyse fonctionelle, 3rd edn (Gauthier-Villars, Paris, 1955).

[23] D. W. Robinson, 'Commutator theory on Hilbert space', Canad. J. Math. 34 (1987), 1235-1280.

[24] D. W. Robinson, Elliptic Operators and Lie Groups, Oxford Mathematical Monographs (Oxford University Press, Oxford, 1991).

[25] D. W. Robinson and A. Sikora, 'Degenerate elliptic operators: capacity, flux and separation', J. Ramanujan Math. Soc. 22 (2007), 385-408.

[26] D. W. Robinson and A. Sikora, 'Degenerate elliptic operators in one dimension', J. Evol. Equ. 10 (2010), 731-759.

[27] D. W. Robinson and A. Sikora, 'Markov uniqueness of degenerate elliptic operators', Ann. Sc. Norm. Super. Pisa Cl. Sci. (2011), to appear, arXiv:0912.4536.

[28] B. Schmuland, 'On the local property for positivity preserving coercive forms', in: Dirichlet Forms and Stochastic Processes, (eds. Z. M. Ma and M. Röckner) (Walter de Gruyter, Berlin, 1995), pp. 345-354. Papers from the International Conference held in Beijing, October 25-31, 1993, and the School on Dirichlet Forms, held in Beijing, October 18-24, 1993.

[29] R. S. Strichartz, 'Analysis of the Laplacian on the complete Riemannian manifold', J. Funct. Anal. 52 (1983), 48-79.

\title{
A. F. M. TER ELST, Department of Mathematics, University of Auckland, Private Bag 92019, Auckland 1142, New Zealand e-mail: terelst@math.auckland.ac.nz
}

\author{
DEREK W. ROBINSON, Mathematical Sciences Institute, \\ Australian National University, Canberra, ACT 0200, Australia \\ e-mail: Derek.Robinson@anu.edu.au
}

\author{
ADAM SIKORA, Department of Mathematics, Macquarie University, \\ Sydney, NSW 2109, Australia \\ e-mail: adam.sikora@mq.edu.au
}

